\title{
ON THE PROPAGATION OF REGULARITY FOR SOLUTIONS OF THE FRACTIONAL KORTEWEG-DE VRIES EQUATION
}

\author{
ARGENIS. J. MENDEZ
}

\begin{abstract}
Aвstract. We consider the initial value problem (IVP) for the fractional Korteweg-de Vries equation (fKdV)

$$
\left\{\begin{array}{l}
\partial_{t} u-D_{x}^{\alpha} \partial_{x} u+u \partial_{x} u=0, \quad x, t \in \mathbb{R}, 0<\alpha<1, \\
u(x, 0)=u_{0}(x)
\end{array}\right.
$$

It has been shown that the solutions to certain dispersive equations satisfy the propagation of regularity phenomena. More precisely, it deals in determine whether regularity of the initial data on the right hand side of the real line is propagated to the left hand side by the flow solution. This property was found originally in solutions of Korteweg-de Vries (KdV) equation and it has been also verified in other dispersive equations as the Benjamin-Ono (BO) equation.

Recently, it has been shown that the solutions of the dispersive generalized BenjaminOno (DGBO) equation, this is $\alpha \in(2,3)$ in $(0.1)$; also satisfy the propagation of regularity phenomena. This is achieved by introducing a commutator decomposition to handle the dispersive part in the equation. Following the approach used in the DGBO, we prove that the solutions of the fKdV also satisfies the propagation of regularity phenomena. Consequently, this type of regularity travels with infinite speed to its left as time evolves.
\end{abstract}

Key words. Fractional KdV. Propagation. Regularity.

\section{InTRODUCTION}

This paper provides a detailed study on the propagation of regularity satisfied by solutions for the so-called fractional Korteweg-de Vries (fKdV) equation

$$
\left\{\begin{array}{l}
\partial_{t} u-D_{x}^{\alpha} \partial_{x} u+u \partial_{x} u=0, \quad x, t \in \mathbb{R}, 0<\alpha<1, \\
u(x, 0)=u_{0}(x)
\end{array}\right.
$$

where $u=u(x, t)$ represents a real valued function and the fractional derivative operator $D_{x}^{s}$ is defined via its Fourier transform as

$$
\widehat{D_{x}^{s} f}(\xi)=c_{s}|\xi|^{s} \hat{f}(\xi) \text { for } s>0
$$

In the case $\alpha=1$, the operator $D_{x}$ can be written as $D_{x}=\mathcal{H} \partial_{x}$ where $\mathcal{H}$ denotes the Hilbert transform,

$$
(\mathcal{H} f)(x)=\frac{1}{\pi} \lim _{\epsilon \rightarrow 0} \int_{|y| \geqslant \epsilon} \frac{f(x-y)}{y} \mathrm{~d} y .
$$

Also, for this particular value of $\alpha$, the equation in (1.1) becomes an integral equation widely studied and known in the literature as the Benjamin-Ono equation (BO) i.e,

$$
\partial_{t} u-\mathcal{H} \partial_{x}^{2} u+u \partial_{x} u=0, \quad x, t \in \mathbb{R} .
$$

The IVP (1.1) has been the focus of attention of recent studies where the local well posedness theory has been approached as well as the existence of solitary wave solutions,

Date: February, 2018

1991 Mathematics Subject Classification. Primary: 35Q53. Secondary: 35Q05.

Key words and phrases. Fractional KdV equation, Well-posedness, Propagation of regularity, Smoothing effect.

This work was partially supported by CNPq, Brazil. 
the stability properties of ground states and numerical simulations, these issues have been largely carried out by Linares et al. [30], [31], Frank and Lenzmann [26], Klein and Saut [25], Molinet, Pilod and Vento [33], and Angulo [35].

The proof of our main result is based on a weighted energy estimate, where the integrability of the term $\partial_{x} u$ is fundamental. So that, for the applicability of this method we will consider local well-posedness (LWP) results in the Sobolev scale with the minimal regularity that ensures $\partial_{x} u \in L^{1}([0, T])$. To our knowledge the best result that fits with our requirements was obtained by Linares, Pilod and Saut [31] when studying weakly dispersive perturbations of Burger's equation. More precisely, they obtained the following:

Theorem 1.2. Let $0<\alpha<1$. Define $s(\alpha):=\frac{3}{2}-\frac{3 \alpha}{8}$ and assume that $s>s(\alpha)$. Then, for every $u_{0} \in H^{S}(\mathbb{R})$, there exists a positive time $T=T\left(\left\|u_{0}\right\|_{s, 2}\right)$ (which can be chosen as a non-increasing functions of its argument) and a unique solution $u$ to (1.1) such that

$$
u \in C\left([0, T]: H^{\mathcal{S}}(\mathbb{R})\right) \quad \text { and } \quad \partial_{x} u \in L^{1}\left([0, T]: L^{\infty}(\mathbb{R})\right) .
$$

It is also important to point out that several improvements have been made in the wellposedness theory of the IVP (1.1). In this sense, we shall mention the recent work announced by Molinet, Pilod and Vento [33], where the authors prove that the IVP (1.1) is locally well-posedness in the Sobolev space $H^{s}(\mathbb{R})$, with $s>\frac{3}{2}-\frac{5 \alpha}{4}$. Additionally, in [33] is proved Global well-posedness (GWP) in the energy space $H^{\alpha / 2}(\mathbb{R})$, as long as $\alpha>\frac{6}{7}$.

Most of these results are based in a method introduced by Molinet and Vento [32] to obtain energy estimates at low regularity for strongly nonresonant dispersive equations. However, the LWP of the IVP (1.1) in the space $H^{s}(\mathbb{R}), s>\frac{3}{2}-\frac{5 \alpha}{4}$, does not ensure the boundeness of $\left\|\partial_{x} u\right\|_{L_{T}^{1} L_{x}^{\infty}}$ which is crucial in our analysis as mentioned above.

Since the space where we will consider solutions has already been described, we will proceed to describe our main result. This is inspired in a property found originally by Isaza, Linares and Ponce [14] in solutions of the KdV. More precisely, the authors prove that regularity on the right hand side of the data travels forward in time with infinite speed. This property "propagation of regularity phenomena principle" was also studied by Isaza et al. [15] in solutions of the Benjamin-Ono equation, where the term that providing dispersion is more difficult to handle due to the presence of the Hilbert transform.

Recently, in [34], we studied the propagation of regularity in solutions of the dispersion generalized Benjamin-Ono equation i.e

$$
\partial_{t} u-D_{x}^{\alpha+1} \partial_{x} u+u \partial_{x} u=0, \quad x, t \in \mathbb{R}, \alpha \in(0,1)
$$

where a combination of the techniques introduced in [14] and [15] together with a commutator decomposition provided by Ginibre and Velo [12]; allowed us to show that real solutions associated to the IVP (1.4) also satisfies the propagation of regularity phenomena. More precisely, this is summarized in the following:

Theorem 1.5. Let $u_{0} \in H^{s}(\mathbb{R})$ with $s=\frac{3-\alpha}{2}$, and $u=u(x, t)$, be the corresponding solution of the IVP (1.4).

If for some $x_{0} \in \mathbb{R}$ and for some $m \in \mathbb{Z}^{+}, m \geqslant 2$,

$$
\partial_{x}^{m} u_{0} \in L^{2}\left(\left\{x \geqslant x_{0}\right\}\right)
$$

then for any $v>0, T>0, \epsilon>0$ and $\tau>4 \epsilon$

$$
\begin{aligned}
& \sup _{0 \leqslant t \leqslant T} \int_{x_{0}+\epsilon-v t}^{\infty}\left(\partial_{x}^{j} u\right)^{2}(x, t) \mathrm{d} x+\int_{0}^{T} \int_{x_{0}+\epsilon-v t}^{x_{0}+\tau-v t}\left(D_{x}^{\frac{\alpha+1}{2}} \partial_{x}^{j} u\right)^{2}(x, t) \mathrm{d} x \mathrm{~d} t \\
& +\int_{0}^{T} \int_{x_{0}+\epsilon-v t}^{x_{0}+\tau-v t}\left(D_{x}^{\frac{\alpha+1}{2}} \mathcal{H} \partial_{x}^{j} u\right)^{2}(x, t) \mathrm{d} x \mathrm{~d} t \leqslant c
\end{aligned}
$$


for $j=1,2, \ldots, m$ with $c=c\left(T ; \epsilon ; v ; \alpha ;\left\|u_{0}\right\|_{H^{s}} ;\left\|\partial_{x}^{m} u_{0}\right\|_{L^{2}\left(\left(x_{0}, \infty\right)\right)}\right)>0$.

If in addition to (1.6) there exists $x_{0} \in \mathbb{R}^{+}$

$$
D_{x}^{\frac{1-\alpha}{2}} \partial_{x}^{m} u_{0} \in L^{2}\left(\left\{x \geqslant x_{0}\right\}\right)
$$

then for any $v \geqslant 0, \epsilon>0$ and $\tau>4 \epsilon$

$$
\begin{aligned}
& \sup _{0 \leqslant t \leqslant T} \int_{x_{0}+\epsilon-v t}^{\infty}\left(D_{x}^{\frac{1-\alpha}{2}} \partial_{x}^{m} u\right)^{2}(x, t) \mathrm{d} x+\int_{0}^{T} \int_{x_{0}+\epsilon-v t}^{x_{0}+\tau-v t}\left(\partial_{x}^{m+1} u\right)^{2}(x, t) \mathrm{d} x \mathrm{~d} t \\
& +\int_{0}^{T} \int_{x_{0}+\epsilon-v t}^{x_{0}+\tau-v t}\left(\partial_{x}^{m+1} \mathcal{H} u\right)^{2}(x, t) \mathrm{d} x \mathrm{~d} t \leqslant c \\
& \text { with } c=c\left(T ; \epsilon ; v ; \alpha ;\left\|u_{0}\right\|_{H^{s}} ;\left\|D_{x}^{\frac{1-\alpha}{2}} \partial_{x}^{m} u_{0}\right\|_{L^{2}\left(\left(x_{0}, \infty\right)\right)}\right)>0 .
\end{aligned}
$$

This property has been found in solutions of several dispersive models, not only in the one dimensional setting, but in the multidimensional one too. In this sense, Isaza et al. [16] proved that real solutions of the Kadomtsev-Petviashvilli (KPII) equation satisfy the propagation of regularity phenomena. Later, Linares and Ponce [29] extended the study to solutions of the Zakharov-Kuznetsov equation in two dimensions resp. three dimensions.

Since this property is present in several dispersive models, as the mentioned above, it seems reasonable to ask if it also satisfied in models with less dispersion than that in DGBO.

So that, a question that arises naturally from the theorem above, is to determine if in the case that less dispersion is considered, for example the IVP (1.1), do the solutions of the $\mathrm{fKdV}$ also satisfy the propagation of regularity phenomena.

Our main objective in this paper is to give answer to this question. More precisely, we obtain that the propagation of regularity phenomena is also satisfied by solutions of the fKdV.

Theorem A. Let $u_{0} \in H^{s_{\alpha}}+(\mathbb{R})$ where $s_{\alpha}:=2-\frac{\alpha}{2}$, and $u=u(x, t)$ be the corresponding solution of the IVP (1.1) provided by Theorem 1.2. Suppose that for some $x_{0} \in \mathbb{R}$ and some $m \in \mathbb{Z}^{+}, m \geqslant 2$,

$$
\partial_{x}^{m} u_{0} \in L^{2}\left(\left\{x \geqslant x_{0}\right\}\right)
$$

Then for any $v \geqslant 0, T>0, \epsilon>0$ and $\tau>4 \epsilon$ are satisfied the following relations:

$$
\begin{aligned}
& \sup _{0 \leqslant t \leqslant T} \int_{x_{0}+\epsilon-v t}^{\infty}\left(\partial_{x}^{j} D_{x}^{\frac{\alpha n}{2}} u\right)^{2}(x, t) \mathrm{d} x+\int_{0}^{T} \int_{x_{0}+\epsilon-v t}^{x_{0}+\tau-v t}\left(D_{x}^{j+\alpha\left(\frac{n+1}{2}\right)} u\right)^{2}(x, t) \mathrm{d} x \mathrm{~d} t \\
& +\int_{0}^{T} \int_{x_{0}+\epsilon-v t}^{x_{0}+\tau-v t}\left(\mathcal{H} D_{x}^{j+\alpha\left(\frac{n+1}{2}\right)} u\right)^{2}(x, t) \mathrm{d} x \mathrm{~d} t \leqslant c, \\
& \quad \sup _{0 \leqslant t \leqslant T} \int_{x_{0}+\epsilon-v t}^{\infty}\left(\partial_{x}^{j} D_{x}^{1-\frac{\alpha}{2}} u\right)^{2}(x, t) \mathrm{d} x+\int_{0}^{T} \int_{x_{0}+\epsilon-v t}^{x_{0}+\tau-v t}\left(\partial_{x}^{j+1} u\right)^{2}(x, t) \mathrm{d} x \mathrm{~d} t \\
& \quad+\int_{0}^{T} \int_{x_{0}+\epsilon-v t}^{x_{0}+\tau-v t}\left(\mathcal{H} \partial_{x}^{j+1} u\right)^{2}(x, t) \mathrm{d} x \mathrm{~d} t \leqslant c
\end{aligned}
$$

and

$$
\begin{aligned}
& \sup _{0 \leqslant t \leqslant T} \int_{x_{0}+\epsilon-v t}^{\infty}\left(\partial_{x}^{m} u\right)^{2}(x, t) \mathrm{d} x+\int_{0}^{T} \int_{x_{0}+\epsilon-v t}^{x_{0}+\tau-v t}\left(D_{x}^{m+\frac{\alpha}{2}} u\right)^{2}(x, t) \mathrm{d} x \mathrm{~d} t \\
& +\int_{0}^{T} \int_{x_{0}+\varepsilon-v t}^{x_{0}+\tau-v t}\left(\mathcal{H} D_{x}^{m+\frac{\alpha}{2}} u\right)^{2}(x, t) \mathrm{d} x \mathrm{~d} t \leqslant c
\end{aligned}
$$

where $j=2,3, \cdots, m-1$ and $n=0,1, \cdots,\left\lceil\frac{2}{\alpha}\right\rceil-1$.

If in addition to (1.10)

$$
D_{x}^{1-\frac{\alpha}{2}} \partial_{x}^{m} u_{0} \in L^{2}\left(\left\{x \geqslant x_{0}\right\}\right) \text { and } D_{x}^{\frac{\alpha j}{2}} \partial_{x}^{m} u_{0} \in L^{2}\left(\left\{x \geqslant x_{0}\right\}\right),
$$




$$
\begin{gathered}
\text { for } j=1,2, \cdots,\left\lceil\frac{2}{\alpha}\right\rceil-1, \text { then for any } v \geqslant 0, T>0, \epsilon>0 \text { and } \tau>4 \epsilon \\
\qquad \begin{array}{c}
\sup _{0 \leqslant t \leqslant T} \int_{\mathbb{R}}\left(\partial_{x}^{m} D_{x}^{\frac{\alpha j}{2}} u\right)^{2}(x, t) \mathrm{d} x+\int_{0}^{T} \int_{\mathbb{R}}\left(D_{x}^{m+\alpha\left(\frac{j+1}{2}\right)} u\right)^{2}(x, t) \mathrm{d} x \mathrm{~d} t \\
+\int_{0}^{T} \int_{\mathbb{R}}\left(\mathcal{H} D_{x}^{m+\alpha\left(\frac{j+1}{2}\right)} u\right)^{2}(x, t) \mathrm{d} x \mathrm{~d} t \leqslant c,
\end{array}
\end{gathered}
$$

and

$$
\begin{aligned}
& \sup _{0 \leqslant t \leqslant T} \int_{x_{0}+\epsilon-v t}^{\infty}\left(\partial_{x}^{m} D_{x}^{1-\frac{\alpha}{2}} u\right)^{2}(x, t) \mathrm{d} x+\int_{0}^{T} \int_{x_{0}+\epsilon-v t}^{x_{0}+\tau-v t}\left(\partial_{x}^{m+1} u\right)^{2}(x, t) \mathrm{d} x \mathrm{~d} t \\
& +\int_{0}^{T} \int_{x_{0}+\epsilon-v t}^{x_{0}+\tau-v t}\left(\mathcal{H} \partial_{x}^{m+1} u\right)^{2}(x, t) \mathrm{d} x \mathrm{~d} t \leqslant c .
\end{aligned}
$$

Remark 1. We shall remark the difference between the index $s(\alpha)$ and $s_{\alpha}$ used in Theorem 1.2 and Theorem A, respectively.

The method of proof used in Theorem A follows in spirit the same lines than that used in the proof of Theorem 1.5. The main differences rely on the inductive argument.

In the case of the DGBO (see (1.4)), the induction is carried out in two steps one for a positive integer $m$ and another for $m+\frac{1-\alpha}{2}, 0<\alpha<1$. In contrast, in the fKdV is required a bi-induction argument, the first for a positive integer $m$, followed by a sequence of steps of the form $m+\alpha j / 2, j=1, \cdots,\left\lceil\frac{2}{\alpha}\right\rceil-1$, where $\lceil\cdot\rceil$ denotes the greatest integer function, and a final one step for $m+1-\alpha / 2$.

Concerning the nonlinear part of the equation in (1.1) the commutator expansion [11], [12] used in studying the propagation of regularity of the DGBO becomes fundamental in our study, as well as the recent advances presented by Li [27] on the study of commutators of Kato-Ponce type.

The document is organized as follows. The first section is focused in the description the notation used thorough all the document. Section 2 is dedicated to present several known results concerning commutator estimates joint with a particular decomposition of these. In section 3 we make a review of the weighted functions later used in the proof of Theorem A. Finally, section 4 deals with the proof of our main result.

\section{Notation}

The following notation will be used extensively throughout this article. The operators $D_{x}^{s}=\left(-\partial_{x}^{2}\right)^{s / 2}$ and $J^{s}=\left(1-\partial_{x}^{2}\right)^{s / 2}$ denotes the Riesz and Bessel potentials of order $-s$, respectively.

For $1 \leqslant p \leqslant \infty, L^{p}(\mathbb{R})$ is the usual Lebesgue space with the norm $\|\cdot\|_{L^{p}}=\|\cdot\|_{p}$, and the besides for $s \in \mathbb{R}$, we consider the Sobolev space $L_{S}^{p}(\mathbb{R})$ is defined via its usual norm $\|f\|_{s, p}=\left\|J^{s} f\right\|_{p}$. In the particular case $p=2$, the set $L_{s}^{2}(\mathbb{R})$ has a Hilbert structure space and we will denote it by $H^{S}(\mathbb{R})$.

Let $f=f(x, t)$ be a function defined for $x \in \mathbb{R}$ and $t$ in the time interval $[0, T]$, with $T>0$ or in the hole line $\mathbb{R}$. Then if $A$ denotes any of the spaces defined above, we define the spaces $L_{T}^{p} A_{x}$ and $L_{t}^{p} A_{x}$ by the norms

$$
\|f\|_{L_{T}^{p} A_{x}}=\left(\int_{0}^{T}\|f(\cdot, t)\|_{A}^{p} \mathrm{~d} t\right)^{1 / p} \quad \text { and }\|f\|_{L_{t}^{p} A_{x}}=\left(\int_{\mathbb{R}}\|f(\cdot, t)\|_{A}^{p} \mathrm{~d} t\right)^{1 / p},
$$

for $1 \leqslant p \leqslant \infty$ with the natural modification in the case $p=\infty$. Moreover, we use similar definitions for the mixed spaces $L_{x}^{q} L_{t}^{p}$ and $L_{x}^{q} L_{T}^{p}$ with $1 \leqslant p, q \leqslant \infty$.

For two quantities $A$ and $B$, we denote $A \lesssim B$ if $A \leqslant c B$ for some constant $c>0$. Similarly $A \gtrsim B$ if $A \geqslant c B$ for some $c>0$. We denote $A \sim B$ if $A \lesssim B$ and $B \lesssim A$. 
The dependence of the constant $c$ on other parameters or constants are usually clear from the context and we will often suppress this dependence.

For $A, B$ operators we will denote the commutator between $A$ and $B$ as $[A ; B]$.

The set of even numbers and odd numbers will be denoted by $\mathbb{Q}_{1}$ and $\mathbb{Q}_{2}$ respectively. Additionally, for $m \in \mathbb{Z}^{+}$the sets $\mathbb{Q}_{1}(m)$ and $\mathbb{Q}_{2}(m)$ will denote the set of even and odd numbers between 1 and $m$, respectively.

\section{INEQUALITIES \& COMMUTATORS}

In this section we collect several inequalities that will be used extensively throughout our work.

First, we have an extension of the Calderon Commutator theorem [6] established by B. Bajšanski et al. [1].

Theorem 3.1. Let $\mathcal{H}$ be the Hilbert transform. Then for any $p \in(1, \infty)$ and any $l, m \in$ $\mathbb{Z}^{+} \cup\{0\}$ there exists $c=c(p ; l ; m)>0$ such that

$$
\left\|\partial_{x}^{l}[\mathcal{H} ; \psi] \partial_{x}^{m} f\right\|_{p} \leqslant c\left\|\partial_{x}^{m+l} \psi\right\|_{\infty}\|f\|_{p} .
$$

The proof follows by results in [1], for a different proof see [[8], Lemma 3.1].

In our analysis, when dealing with the nonlinear part of the equation (1.1); it will be crucial the next inequalities concerning the Leibniz rule for fractional derivatives established in $[13,19,22]$.

Lemma 3.1. For $s>0, p \in[1, \infty)$

$$
\left\|D^{s}(f g)\right\|_{p} \lesssim\|f\|_{p_{1}}\left\|D^{s} g\right\|_{p_{2}}+\|g\|_{p_{3}}\left\|D^{s} f\right\|_{p_{4}}
$$

with

$$
\frac{1}{p}=\frac{1}{p_{1}}+\frac{1}{p_{2}}=\frac{1}{p_{3}}+\frac{1}{p_{4}}, \quad p_{j} \in(1, \infty], \quad j=1,2,3,4 .
$$

Recently D.Li [27] proved new fractional Leibniz rules for the nonlocal operator $D^{s}, s>$ 0 , and related ones, including various end-point situations. This type of estimate has proved to be a very useful tool in the study of propagation of regularity.

Theorem 3.4. Case 1: $1<p<\infty$.

Let $s>0$ and $1<p<\infty$. Then for any $s_{1}, s_{2} \geqslant 0$ with $s=s_{1}+s_{2}$, and any $f, g \in \mathcal{S}(\mathbb{R})$, the following hold:

(1) If $1<p_{1}, p_{2}<\infty$ with $\frac{1}{p}=\frac{1}{p_{1}}+\frac{1}{p_{2}}$, then

$$
\left\|D^{s}(f g)-\sum_{\alpha \leqslant s_{1}} \frac{1}{\alpha !} \partial_{x}^{\alpha} f D^{s, \alpha} g-\sum_{\beta \leqslant s_{2}} \frac{1}{\beta !} \partial_{x}^{\beta} g D^{s, \beta} f\right\|_{p} \lesssim\left\|D^{s_{1}} f\right\|_{p_{1}}\left\|D^{s_{2}} g\right\|_{p_{2}} .
$$

(2) If $p_{1}=p, p_{2}=\infty$, then

$$
\left\|D^{s}(f g)-\sum_{\alpha<s_{1}} \frac{1}{\alpha !} \partial_{x}^{\alpha} f D^{s, \alpha} g-\sum_{\beta \leqslant s_{2}} \frac{1}{\beta !} \partial_{x}^{\beta} g D^{s^{\prime} \beta} f\right\|_{p} \lesssim\left\|D^{s_{1}} f\right\|_{p}\left\|D^{s_{2}} g\right\|_{\mathrm{BMO}},
$$

where $\|\cdot\|_{\mathrm{BMO}}$ denotes the norm in the BMO space

(3) If $p_{1}=\infty, p_{2}=p$, then

$$
\left\|D^{s}(f g)-\sum_{\alpha \leqslant s_{1}} \frac{1}{\alpha !} \partial^{\alpha} f D^{s, \alpha} g-\sum_{\beta<s_{2}} \frac{1}{\beta !} \partial^{\beta} g D^{s, \beta} f\right\|_{p} \lesssim\left\|D^{s_{1}} f\right\|_{\mathrm{BMO}}\left\|D^{s_{2}} g\right\|_{p} .
$$


The operator $D^{s, \alpha}$ is defined via Fourier transform ${ }^{1}$

$$
\begin{aligned}
& \widehat{D^{s, \alpha} g}(\xi)=\widehat{D^{s, \alpha}}(\xi) \widehat{g}(\xi), \\
& \widehat{D^{s, \alpha}}(\xi)=i^{-\alpha} \partial_{\xi}^{\alpha}\left(|\xi|^{s}\right) .
\end{aligned}
$$

Case 2: $\frac{1}{2}<p \leqslant 1$.

If $\frac{1}{2}<p \leqslant 1, s>\frac{1}{p}-1$ or $s \in 2 \mathbb{N}$, then for any $1<p_{1}, p_{2}<\infty$ with

$$
\frac{1}{p}=\frac{1}{p_{1}}+\frac{1}{p_{2}}
$$

any $s_{1}, s_{2} \geqslant 0$ with $s_{1}+s_{2}=s$,

$$
\left\|D^{s}(f g)-\sum_{\alpha \leqslant s_{1}} \frac{1}{\alpha !} \partial_{x}^{\alpha} f D^{s, \alpha} g-\sum_{\beta \leqslant s_{2}} \frac{1}{\beta !} \partial_{x}^{\beta} g D^{s, \beta} f\right\|_{p} \lesssim\left\|D^{s_{1}} f\right\|_{p_{1}}\left\|D^{s_{2}} g\right\|_{p_{2}} .
$$

Remark 2. As usual empty summation (such as $\sum_{0 \leqslant \alpha<0}$ ) is defined as zero.

Proof. For a detailed proof of this theorem and related results, see [27].

Also, several commutator estimates have been obtained by D. Li [27]. These, corresponds to a family of refined Kato-Ponce type inequalities for the operator $D^{s}$.

Lemma 3.2. Let $1<p<\infty$. Let $1<p_{1}, p_{2}, p_{3}, p_{4} \leqslant \infty$ satisfy

$$
\frac{1}{p}=\frac{1}{p_{1}}+\frac{1}{p_{2}}=\frac{1}{p_{3}}+\frac{1}{p_{4}}
$$

Therefore,

(a) If $0<s \leqslant 1$, then

$$
\left\|D^{s}(f g)-f D^{s} g\right\|_{p} \lesssim\left\|D^{s-1} \partial_{x} f\right\|_{p_{1}}\|g\|_{p_{2}} .
$$

(b) If $s>1$, then

$$
\left\|D^{s}(f g)-f D^{s} g\right\|_{p} \lesssim\left\|D^{s-1} \partial_{x} f\right\|_{p_{1}}\|g\|_{p_{2}}+\left\|\partial_{x} f\right\|_{p_{3}}\left\|D^{s-1} g\right\|_{p_{4}} .
$$

In addition, a non sharp commutator estimate is required in our analysis.

Lemma 3.3. Let $\phi \in C^{\infty}(\mathbb{R})$ with $\phi^{\prime} \in C_{0}^{\infty}(\mathbb{R})$. If $f \in H^{s}(\mathbb{R}), s>0$, then for any $l>s+\frac{1}{2}$

$$
\left\|\left[D_{x}^{s} ; \phi\right] f\right\|_{2} \lesssim\left\|\phi^{\prime}\right\|_{l, 2}\|f\|_{s-1,2} .
$$

Proof. We will give an outline of the proof.

First, we use the decomposition of the operator $D_{x}^{s}$ for $s>0$, given by Bourgain and $\mathrm{Li}$ [5] in the study of commutator estimates.

In fact,

$$
D_{x}^{s}=J_{x}^{s}-\sum_{1 \leqslant j \leqslant \frac{s}{2}} c_{s, j} j_{x}^{s-2 j}+K_{s}
$$

where $K_{s}$ is a bounded integral operator satisfying $K_{s}: L^{p}(\mathbb{R}) \longrightarrow L^{p}(\mathbb{R}), 1 \leqslant p \leqslant \infty$.

By using this decomposition and a proof similar to that found in $[9$, Chapter 6 , Lemma 6.16 ], the lemma follows.

Also, the following inequality of Gagliardo-Nirenberg type is used.

\footnotetext{
${ }^{1}$ The precise form of the Fourier transform does not matter.
} 
Lemma 3.4. Let $1<q, p<\infty, 1<r \leqslant \infty$ and $0<\alpha<\beta$. Then,

$$
\left\|D^{\alpha} f\right\|_{L^{p}} \lesssim c\|f\|_{L^{r}}^{1-\theta}\left\|D^{\beta} f\right\|_{L^{q}}^{\theta}
$$

with

$$
\frac{1}{p}-\alpha=(1-\theta) \frac{1}{r}+\theta\left(\frac{1}{q}-\beta\right), \quad \theta \in[\alpha / \beta, 1] .
$$

Proof. See [3, chapter 4].

Next we consider a result that will be used widely when dealing with the nonlinear part of the fKdV.

Lemma 3.5. Let $m \in \mathbb{Z}^{+}$and $s \geqslant 0$. If $f \in L^{2}(\mathbb{R})$ and $g \in L^{p}(\mathbb{R}), 2 \leqslant p \leqslant \infty$, with

$$
\operatorname{dist}(\operatorname{supp}(f), \operatorname{supp}(g)) \geqslant \delta>0 \text {. }
$$

Then

$$
\left\|g \partial_{x}^{m} D^{s} f\right\|_{L^{p}} \lesssim\|g\|_{L^{p}}\|f\|_{L^{2}}
$$

Remark 3. A previous estimated was obtained by Kenig et al. [20] when studying the propagation of regularity (fractional case) but for the operator $J^{s}$.

Proof. See [34, Lemma 3.28].

3.1. Commutator Expansions. In this section we present a several auxiliary results obtained by Ginibre and Velo [11], [12] which has proved to be useful in the study of propagation of regularity.

Let $a=2 \mu+1>1$, let $n$ be a nonnegative integer and $f$ be a smooth function with suitable decay at infinity, for instance with $f^{\prime} \in C_{0}^{\infty}(\mathbb{R})$.

We define the operator

$$
R_{n}(a)=-\left[\mathcal{H} D^{a} ; f\right]-\frac{1}{2}\left(P_{n}(a)-\mathcal{H} P_{n}(a) \mathcal{H}\right),
$$

where

$$
P_{n}(a)=a \sum_{0 \leqslant j \leqslant n} c_{2 j+1}(-1)^{j} 4^{-j} D^{\mu-j} f^{(2 j+1)} D^{\mu-j},
$$

and the constants $c_{2 j+1}$ are given by the following formula

$$
c_{1}=1 \quad \text { and } \quad c_{2 j+1}=\frac{1}{(2 j+1) !} \prod_{0 \leqslant k<j}\left(a^{2}-(2 k+1)^{2}\right) .
$$

Proposition 3.1. Let $n$ be a non-negative integer, $a \geqslant 1$, and $\sigma \geqslant 0$, be such that

$$
2 n+1 \leqslant a+2 \sigma \leqslant 2 n+3 .
$$

Then

(a) The operator $D^{\sigma} R_{n}(a) D^{\sigma}$ is bounded in $L^{2}$ with norm

$$
\left\|D^{\sigma} R_{n}(a) D^{\sigma} f\right\|_{2} \leqslant C(2 \pi)^{-1 / 2}\left\|\left(\widehat{D^{a+2 \sigma}} f\right)\right\|_{1}\|f\|_{2} .
$$

If $a \geqslant 2 n+1$, one can take $C=1$.

(b) Assume in addition that

$$
2 n+1 \leqslant a+2 \sigma<2 n+3 .
$$

Then the operator $D^{\sigma} R_{n}(a) D^{\sigma}$ is compact in $L^{2}(\mathbb{R})$.

Proof. See Proposition 2.2 in [12]. 
Remark 4. Proposition 3.1 is a generalization from previous results, where the derivatives of operator $R_{n}(a)$ are not consider (cf. Proposition 1 in [11]).

Also, a direct application of the commutator decomposition(3.12) is the smoothing effect associated to solutions of the IVP (1.1).

Proposition 3.2. Let $\varphi$ denote a nondecreasing smooth function such that $\operatorname{supp} \varphi^{\prime} \subset$ $(-1,2)$ and $\left.\varphi\right|_{[0,1]} \equiv 1$. For $j \in \mathbb{Z}$, we define $\varphi_{j}(x)=\varphi(x-j)$. Let $u \in C\left([0, T]: H^{\infty}(\mathbb{R})\right)$ be a smooth solution of (1.1) with $0<\alpha<1$. Assume also that $s \geqslant 0$ and $r>\frac{1}{2}$. Then,

$$
\begin{aligned}
& \left(\int_{0}^{T} \int_{\mathbb{R}}\left(\left|D_{x}^{s+\frac{\alpha}{2}} u(x, t)\right|^{2}+\left|\mathcal{H} D_{x}^{s+\frac{\alpha}{2}} u(x, t)\right|^{2}\right) \varphi_{j}^{\prime}(x) \mathrm{d} x \mathrm{~d} t\right)^{1 / 2} \\
& \lesssim\left(1+T+\left\|\partial_{x} u\right\|_{L_{T}^{1} L_{x}^{\infty}}+T\|u\|_{L_{T}^{\infty} H_{x}^{r}}\right)^{1 / 2}\|u\|_{L_{T}^{\infty} H_{x}^{s} .}
\end{aligned}
$$

Proof. As was mentioned above the proof use the decomposition (3.12) and a application of Mikhlin's Theorem. For a detailed description of the proof see [31, Proposition 2.12].

Remark 5. In the particular case that $u_{0}$ is in the Sobolev space $H^{s_{\alpha}}(\mathbb{R}), s_{\alpha}=2-\frac{\alpha}{2}$, the gain of local derivatives is summarized in the following inequality

$$
\left(\int_{0}^{T} \int_{-r}^{r}\left(\left|\partial_{x}^{2} u(x, t)\right|^{2}+\left|\mathcal{H} \partial_{x}^{2} u(x, t)\right|^{2}\right) \mathrm{d} x \mathrm{~d} t\right)^{1 / 2} \leqslant C\left(r ; T ;\left\|u_{0}\right\|_{s_{\alpha}, 2}\right)
$$

for any $r>0$. The proof follows combining the ideas used in the proof of Proposition 3.2 and the inequality

$$
\|u\|_{L_{T}^{\infty} H_{x}^{s \alpha}} \lesssim\left\|u_{0}\right\|_{H_{x}^{s}} \mathrm{e}^{\left\|\partial_{x} u\right\|_{L_{T}^{1} L_{x}^{\infty}}}
$$

The last inequality above is widely know in the literature and its proof is based on energy estimate combined with commutator estimates Kato-Ponce type. For a detailed proof see for instance [28, Chapter 9, p. 221]

\section{Weighted Functions}

This section is devoted to describe the the weighted functions used in the proof of Theorem A, as well as their properties.

Most of these functions where originally constructed when studying the propagation of regularity phenomena for solutions of the KdV [14], [20].

For $\epsilon>0$ and $b \geqslant 5 \epsilon$ define the families of functions

$$
\chi_{\epsilon, b}, \phi_{\epsilon, b}, \widetilde{\phi_{\epsilon}, b}, \psi_{\epsilon}, \eta_{\epsilon, b}, \varphi_{\epsilon, b} \in C^{\infty}(\mathbb{R})
$$

satisfying the following properties:

(1) $\chi_{\epsilon, b}^{\prime} \geqslant 0$,

(2) $\chi_{\epsilon, b}(x)= \begin{cases}0, & x \leqslant \epsilon \\ 1, & x \geqslant b,\end{cases}$

(3) $\operatorname{supp}\left(\chi_{\epsilon, b}\right) \subseteq[\epsilon, \infty)$;

(4) $\chi_{\epsilon, b}^{\prime}(x) \geqslant \frac{1}{10(b-\epsilon)} \mathbb{1}_{[2 \epsilon, b-2 \epsilon]}(x)$,

(5) $\operatorname{supp}\left(\chi_{\epsilon, b}^{\prime}\right) \subseteq[\epsilon, b]$.

(6) There exists real numbers $c_{j}$ such that

$$
\left|\chi_{\epsilon, b}^{(j)}(x)\right| \leqslant c_{j} \chi_{\epsilon / 3, b+\epsilon}^{\prime}(x), \quad \forall x \in \mathbb{R}, j \in \mathbb{Z}^{+} .
$$


(7) For $x \in(3 \epsilon, \infty)$

$$
\chi_{\epsilon, b}(x) \geqslant \frac{1}{2} \frac{\epsilon}{b-3 \epsilon} .
$$

(8) For $x \in \mathbb{R}$

$$
\chi_{\epsilon / 3, b+\epsilon}^{\prime}(x) \leqslant \frac{\epsilon}{b-3 \epsilon} .
$$

(9) Also, given $\epsilon>0$ and $b \geqslant 5 \epsilon$ there exist $c_{1}, c_{2}>0$ such that

$$
\begin{aligned}
& \chi_{\epsilon, b}^{\prime}(x) \leqslant c_{1} \chi_{\epsilon / 3, b+\epsilon}^{\prime}(x) \chi_{\epsilon / 3, b+\epsilon}(x), \\
& \chi_{\epsilon, b}^{\prime}(x) \leqslant c_{2} \chi_{\epsilon / 5, \epsilon}(x) .
\end{aligned}
$$

(10) For $\epsilon>0$ given and $b \geqslant 5 \epsilon$, we define the functions

$$
\eta_{\epsilon, b}=\sqrt{\chi_{\epsilon, b} \chi_{\epsilon, b}^{\prime}} \text { and } \varphi_{\epsilon, b}=\sqrt{\chi_{\epsilon, b}^{\prime}} .
$$

(11) $\operatorname{supp}\left(\phi_{\epsilon, b}\right), \operatorname{supp}\left(\widetilde{\phi_{\epsilon, b}}\right) \subset[\epsilon / 4, b]$,

(12) $\phi_{\epsilon}(x)=\widetilde{\phi_{\epsilon, b}}(x)=1, \quad x \in[\epsilon / 2, \epsilon]$,

(13) $\operatorname{supp}\left(\psi_{\epsilon}\right) \subseteq(-\infty, \epsilon / 2]$,

(14) for $x \in \mathbb{R}$

$$
\chi_{\epsilon, b}(x)+\phi_{\epsilon, b}(x)+\psi_{\epsilon}(x)=1,
$$

and

$$
\chi_{\epsilon, b}^{2}(x)+{\widetilde{\phi_{\epsilon, b}}}^{2}(x)+\psi_{\epsilon}(x)=1 .
$$

The family $\left\{\chi_{\epsilon, b}: \epsilon>0, b \geqslant 5 \epsilon\right\}$ is constructed as follows: let $\rho \in C_{0}^{\infty}(\mathbb{R}), \rho(x) \geqslant 0$, even, with $\operatorname{supp}(\rho) \subseteq(-1,1)$ and $\|\rho\|_{1}=1$.

Then defining

$$
v_{\epsilon, b}(x)= \begin{cases}0, & x \leqslant 2 \epsilon \\ \frac{x}{b-3 \epsilon}-\frac{2 \epsilon}{b-3 \epsilon}, & 2 \epsilon \leqslant x \leqslant b-\epsilon \\ 1, & x \geqslant b-\epsilon\end{cases}
$$

and

$$
\chi_{\epsilon, b}(x)=\rho_{\epsilon} * v_{\epsilon, b}(x)
$$

where $\rho_{\epsilon}(x)=\epsilon^{-1} \rho(x / \epsilon)$.

\section{Proof of Theorem A}

We shall use two induction arguments. One for $m \in \mathbb{Z}^{+}$with $m \geqslant 2$ and the other one for $m+\alpha k$, where $k$ is a positive integer to be specified later in the proof.

Since the solutions of the IVP (1.1) are translation invariant, then without loss of generality we will take $x_{0}=0$.

Also, it will be assumed that solutions of the IVP (1.1) are real valued functions with as much regularity as required.

\section{$\underline{\text { CASE } m=2:}$}

STEP 1:

Formally, we apply $\partial_{x}^{2}$ to the equation in (1.1) followed by a multiplication by $\partial_{x}^{2} u \chi_{\epsilon, b}^{2}$ to obtain

$$
\partial_{x}^{2} \partial_{t} u \partial_{x}^{2} u \chi_{\epsilon, b}^{2}-\partial_{x}^{2} D_{x}^{\alpha} \partial_{x} u \partial_{x}^{2} u \chi_{\epsilon, b}^{2}+\partial_{x}^{2}\left(u \partial_{x} u\right) \partial_{x}^{2} u \chi_{\epsilon, b}^{2}=0
$$


after integrating in the position variable, we obtain the energy identity

$$
\begin{aligned}
& \frac{1}{2} \frac{\mathrm{d}}{\mathrm{d} t} \int_{\mathbb{R}}\left(\partial_{x}^{2} u\right)^{2} \chi_{\epsilon, b}^{2} \mathrm{~d} x-\underbrace{\frac{v}{2} \int_{\mathbb{R}}\left(\partial_{x}^{2} u\right)^{2}}_{B_{1}(t)}-\underbrace{\left(\chi_{\epsilon, b}^{2}\right)^{\prime} \mathrm{d} x}_{B_{2}(t)}-\underbrace{\underbrace{\int_{\mathbb{R}}\left(\partial_{x}^{2} D_{x}^{\alpha} \partial_{x} u\right)}_{\mathbb{R}}}_{B_{3}(t)} \\
& +\underbrace{\partial_{x}^{2} u \chi_{\epsilon, b}^{2} \mathrm{~d} x}_{\underbrace{\int_{x} \partial_{x}^{2}\left(u \partial_{x} u\right)}_{\mathbb{R}}}=0 .
\end{aligned}
$$

$\S .1$ To handle $B_{1}$ first notice that there exists $c>0$ and $r>0$ such that

$$
\left(\chi_{\epsilon, b}^{2}\right)^{\prime}(x+v t)=2 \chi_{\epsilon, b}(x+v t) \chi_{\epsilon, b}^{\prime}(x+v t) \leqslant c \mathbb{1}_{[-r, r]}(x)
$$

for all $(x, t) \in \mathbb{R} \times[0, T]$. Thus, by Remark 3.18 , it follows that

$$
\begin{aligned}
\int_{0}^{T}\left|B_{1}(t)\right| \mathrm{d} t & \lesssim \int_{0}^{T} \int_{\mathbb{R}}\left(\partial_{x}^{2} u\right)^{2}\left(\chi_{\epsilon, b}^{2}\right)^{\prime} \mathrm{d} x \mathrm{~d} t \\
& \lesssim \int_{0}^{T} \int_{-r}^{r}\left(\partial_{x}^{2} u\right)^{2} \mathrm{~d} x \mathrm{~d} t \\
& \leqslant c\left(\left\|u_{0}\right\|_{s_{\alpha}, 2} ; r ; T\right) .
\end{aligned}
$$

$\S .2$ Now, we extract information from the term handling the dispersive part of the equation in (1.1).

Combining integration by parts and Plancherel's identity it follows that

$$
\begin{aligned}
B_{2}(t) & =-\frac{1}{2} \int_{\mathbb{R}} \partial_{x}^{2} u\left[\mathcal{H} D_{x}^{\alpha+1} ; \chi_{\epsilon, b}\right] \partial_{x}^{2} u \mathrm{~d} x \\
& =-\frac{1}{2} \int_{\mathbb{R}} D_{x}^{2} u\left[\mathcal{H} D_{x}^{\alpha+1} ; \chi_{\epsilon, b}\right] D_{x}^{2} u \mathrm{~d} x
\end{aligned}
$$

Then using (3.12)

$$
\left[\mathcal{H} D_{x}^{\alpha+1} ; \chi_{\epsilon, b}\right]=-R_{n}(\alpha+1)-\frac{1}{2} P_{n}(\alpha+1)+\frac{1}{2} \mathcal{H} P_{n}(\alpha+1) \mathcal{H}
$$

for some nonnegative integer $n$ to be fixed. This will help to obtain the smoothing effect. Indeed, replacing this decomposition into $B_{2}$ yields

$$
\begin{aligned}
B_{2}(t)= & \frac{1}{2} \int_{\mathbb{R}} D_{x}^{2} u R_{n}(\alpha+1) D_{x}^{2} u \mathrm{~d} x+\frac{1}{4} \int_{\mathbb{R}} D_{x}^{2} u P_{n}(\alpha+1) D_{x}^{2} u \mathrm{~d} x \\
& -\frac{1}{4} \int_{\mathbb{R}} D_{x}^{2} u \mathcal{H} P_{n}(\alpha+1) \mathcal{H} D_{x}^{2} u \mathrm{~d} x \\
= & B_{2,1}(t)+B_{2,2}(t)+B_{2,3}(t) .
\end{aligned}
$$

The quantity of terms $n$, will be chosen according to the following rule (see (3.15))

$$
2 n+1 \leqslant \alpha+5 \leqslant 2 n+3,
$$

which clearly implies $n=2$.

In view of (3.16) the remainder operator $R_{2}(\alpha+1)$ satisfies

$$
\left\|D_{x}^{2} R_{2}(\alpha+1) D_{x}^{2} f\right\|_{2} \lesssim\|f\|_{2}\left\|D_{x}^{\alpha+5\left(\chi_{\epsilon, b}^{2}\right)}\right\|_{1}{ }^{\prime}
$$

for $f$ in a suitable class of functions. 
For our proposes a combination of (5.5) with Hölder's inequality and Plancherel's identity is sufficient to obtain

$$
B_{2,1}(t)=\frac{1}{2} \int_{\mathbb{R}} u D_{x}^{2} R_{2}(\alpha+1) D_{x}^{2} u \mathrm{~d} x \lesssim\left\|u_{0}\right\|_{2}^{2}\left\|\widehat{D_{x}^{\alpha+5}\left(\chi_{\epsilon, b}^{2}\right)}\right\|_{1} .
$$

Therefore, integrating in time yields

$$
\int_{0}^{T}\left|B_{2,1}(t)\right| \mathrm{d} t \leqslant c
$$

Replacing $P_{2}(\alpha+1)$ into $B_{2,2}$ and $B_{2,3}$ produce

$$
\begin{aligned}
B_{2,2}(t)= & \left(\frac{\alpha+1}{4}\right) \int_{\mathbb{R}}\left(D_{x}^{2+\frac{\alpha}{2}} u\right)^{2}\left(\chi_{\epsilon, b}^{2}\right)^{\prime} \mathrm{d} x-c_{3}\left(\frac{\alpha+1}{16}\right) \int_{\mathbb{R}}\left(D_{x}^{1+\frac{\alpha}{2}} u\right)^{2}\left(\chi_{\epsilon, b}^{2}\right)^{(3)} \mathrm{d} x \\
& +c_{5}\left(\frac{\alpha+1}{64}\right) \int_{\mathbb{R}}\left(D_{x}^{\frac{\alpha}{2}} u\right)^{2}\left(\chi_{\epsilon, b}^{2}\right)^{(5)} \mathrm{d} x \\
= & B_{2,2,1}(t)+B_{2,2,2}(t)+B_{2,2,3}(t) .
\end{aligned}
$$

and

$$
\begin{aligned}
B_{2,3}(t) & =\left(\frac{\alpha+1}{4}\right) \int_{\mathbb{R}}\left(\mathcal{H} D_{x}^{2+\frac{\alpha}{2}} u\right)^{2}\left(\chi_{\epsilon, b}^{2}\right)^{\prime} \mathrm{d} x \\
& -c_{3}\left(\frac{\alpha+1}{16}\right) \int_{\mathbb{R}}\left(\mathcal{H} D_{x}^{1+\frac{\alpha}{2}} u\right)^{2}\left(\chi_{\epsilon, b}^{2}\right)^{(3)} \mathrm{d} x+c_{5}\left(\frac{\alpha+1}{64}\right) \int_{\mathbb{R}}\left(\mathcal{H} D_{x}^{\frac{\alpha}{2}} u\right)^{2}\left(\chi_{\epsilon, b}^{2}\right)^{(5)} \mathrm{d} x \\
& =B_{2,3,1}(t)+B_{2,3,2}(t)+B_{2,3,3}(t) .
\end{aligned}
$$

Notice that $B_{2,2,1}$ and $B_{2,3,1}$ are positive and represent the smoothing effect. Then we need to bound the terms $B_{2,2,2}, B_{2,2,3}, B_{2,3,2}$, and $B_{2,3,3}$.

After integrate in time the terms $B_{2,2,2}, B_{2,3,2}, B_{2,2,3}$, and $B_{2,3,3}$ can be handled by using the local theory i.e.

$$
\int_{0}^{T}\left|B_{2,2+l, 2}(t)\right| \mathrm{d} t \lesssim \sup _{0 \leqslant t \leqslant T}\|u(t)\|_{s_{\alpha}, 2} \quad \text { for } \quad l \in\{0,1\},
$$

and

$$
\int_{0}^{T}\left|B_{2,2+l, 3}(t)\right| \mathrm{d} t \lesssim \sup _{0 \leqslant t \leqslant T}\|u(t)\|_{s_{\alpha}, 2} \quad \text { for } \quad l \in\{0,1\}
$$

§.3 Finally, we handle $B_{3}$ firstly applying integration by parts as follows

$$
\begin{aligned}
B_{3}(t) & =\frac{5}{2} \int_{\mathbb{R}} \partial_{x} u\left(\partial_{x}^{2} u\right)^{2} \chi_{\epsilon, b}^{2} \mathrm{~d} x-\frac{1}{2} \int_{\mathbb{R}} u\left(\partial_{x}^{2} u\right)^{2}\left(\chi_{\epsilon, b}^{2}\right)^{\prime} \mathrm{d} x \\
& =B_{3,1}(t)+B_{3,2}(t) .
\end{aligned}
$$

Since $u$ satisfies Strichartz estimate i.e. $\partial_{x} u \in L^{1}\left([0, T]: L^{\infty}(\mathbb{R})\right)$ in Theorem 1.2, then

$$
\left|B_{3,1}(t)\right| \lesssim\left\|\partial_{x} u(t)\right\|_{\infty} \int_{\mathbb{R}}\left(\partial_{x}^{2} u\right)^{2} \chi_{\epsilon, b}^{2} \mathrm{~d} x
$$

We shall also point out that the integral expression on the right hand side of (5.6) will be estimated by using Gronwall's inequality. 
An application of Sobolev's embedding produces

$$
\begin{aligned}
\left|B_{3,2}(t)\right| & \lesssim\|u(t)\|_{\infty} \int_{\mathbb{R}}\left(\partial_{x}^{2} u\right)^{2}\left(\chi_{\epsilon, b}^{2}\right)^{\prime} \mathrm{d} x \\
& \lesssim \sup _{0 \leqslant t \leqslant T}\|u(t)\|_{s_{\alpha}, 2} \int_{\mathbb{R}}\left(\partial_{x}^{2} u\right)^{2}\left(\chi_{\epsilon, b}^{2}\right)^{\prime} \mathrm{d} x
\end{aligned}
$$

We finish this step gathering the estimates above, this together with an application of Gronwall's inequality yield

$$
\sup _{0 \leqslant t \leqslant T}\left\|\partial_{x}^{2} u \chi_{\epsilon, b}(\cdot+v t)\right\|_{2}^{2}+\left\|D_{x}^{2+\frac{\alpha}{2}} u \eta_{\epsilon, b}^{2}\right\|_{L_{T}^{2} L_{x}^{2}}^{2}+\left\|\mathcal{H} D_{x}^{2+\frac{\alpha}{2}} u \eta_{\epsilon, b}^{2}\right\|_{L_{T}^{2} L_{x}^{2}}^{2} \leqslant c_{2,1}^{*}
$$

for any $\epsilon>0, b \geqslant 5 \epsilon$ and $v \geqslant 0$.

At this point several issues shall be clarified and fixed. First, we indicate the dependence on the parameters involved in the constant $c_{2,1}^{*}$, this will be crucial later when we will consider the limit process. More precisely, $c_{2,1}^{*}=c_{2,1}^{*}\left(\alpha ; \epsilon ; T ; v ;\left\|u_{0}\right\|_{s_{\alpha}, 2} ;\left\|\partial_{x}^{2} u_{0} \chi_{\epsilon, b}\right\|_{2}\right)$.

These families of constants are distinguished in our argument, so that we will differentiate them by fixing the following notation: for $m, n \in \mathbb{Z}^{+}$, the number $c_{m, n}^{*}$ corresponds to the case of $m$-derivatives in the $n$th step of the induction process.

Without more delays we proceed to the case 2 in our inductive process. This step is summarized in the following diagram

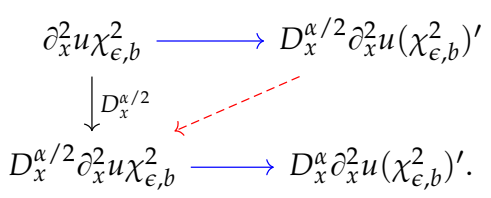

The columns on the left hand side indicates the propagation of regularity, which is carried out by steps of length $\alpha / 2$, except for the final step that will be exemplified later. Instead, the columns on the right hand side furnish the smoothing effect obtained at that level of propagation.

Finally, the diagonal lines are to indicate the dependence of the smoothing effect in the next level of propagation.

Now that the strategy has been explained we proceed to estimate.

STEP 2:

After apply the operator $D_{x}^{\frac{\alpha}{2}} \partial_{x}^{2}$ to the equation in (1.1) and multiply the resulting equation by $D_{x}^{\frac{\alpha}{2}} \partial_{x}^{2} u \chi_{\epsilon, b}^{2}(x+v t)$ one gets

$$
\begin{aligned}
& \frac{1}{2} \frac{\mathrm{d}}{\mathrm{d} t} \int_{\mathbb{R}}\left(\partial_{x}^{2} D_{x}^{\frac{\alpha}{2}} u\right)^{2} \chi_{\epsilon, b}^{2} \mathrm{~d} x-\underbrace{\frac{v}{2} \int_{\mathbb{R}}\left(\partial_{x}^{2} D_{x}^{\frac{\alpha}{2}} u\right)^{2}\left(\chi_{\epsilon, b}^{2}\right)^{\prime} \mathrm{d} x} \\
& B_{1}(t) \\
& -\underbrace{\int_{\mathbb{R}}\left(\partial_{x}^{2} D_{x}^{\frac{\alpha}{2}} D_{x}^{\alpha} \partial_{x} u\right) \partial_{x}^{2} D_{x}^{\frac{\alpha}{2}} u \chi_{\epsilon, b}^{2} \mathrm{~d} x}_{B_{2}(t)}+\underbrace{\int_{\mathbb{R}}\left(\partial_{x}^{2} D_{x}^{\frac{\alpha}{2}}\left(u \partial_{x} u\right)\right) \partial_{x}^{2} D_{x}^{\frac{\alpha}{2}} u \chi_{\epsilon, b}^{2} \mathrm{~d} x}_{B_{3}(t)}=0 .
\end{aligned}
$$


$\S .1$ As we indicated in the diagram above, to handle $\left\|B_{1}\right\|_{1}$ it is only required to use (5.8). Then,

$$
\int_{0}^{T}\left|B_{1}(t)\right| \mathrm{d} t \leqslant \frac{|v|}{2} \int_{0}^{T} \int_{\mathbb{R}}\left(\partial_{x}^{2} D_{x}^{\frac{\alpha}{2}} u\right)^{2}\left(\chi_{\epsilon, b}^{2}\right)^{\prime} \mathrm{d} x \mathrm{~d} t \leqslant c_{2,1}^{*}
$$

$\S .2$ The term $B_{2}$ shall be rewritten as was done before in order to obtain the corresponding smoothing effect in this step. Thus, we apply integration by parts and Plancherel's identity to get

$$
B_{2}(t)=-\frac{1}{2} \int_{\mathbb{R}} D_{x}^{2+\frac{\alpha}{2}} u\left[\mathcal{H} D_{x}^{\alpha+1} ; \chi_{\epsilon, b}^{2}\right] D_{x}^{2+\frac{\alpha}{2}} u \mathrm{~d} x
$$

By using the commutator decomposition (5.4) into (5.10)we have

$$
\begin{aligned}
B_{2}(t)= & \frac{1}{4} \int_{\mathbb{R}} D_{x}^{2+\frac{\alpha}{2}} u R_{n}(\alpha+1) D_{x}^{2+\frac{\alpha}{2}} u \mathrm{~d} x+\frac{1}{4} \int_{\mathbb{R}} D_{x}^{2+\frac{\alpha}{2}} u P_{n}(\alpha+1) D_{x}^{2+\frac{\alpha}{2}} u \mathrm{~d} x \\
& -\frac{1}{4} \int_{\mathbb{R}} D_{x}^{2+\frac{\alpha}{2}} u \mathcal{H} P_{n}(\alpha+1) \mathcal{H} D_{x}^{2+\frac{\alpha}{2}} u \mathrm{~d} x \\
= & B_{2,1}(t)+B_{2,2}(t)+B_{2,3}(t),
\end{aligned}
$$

for some positive integer $n$ to be fixed.

From this term we obtain the smoothing effect by using a similar analysis as the used in the previous step. This argument allow us to fix $n=2$, and for this particular value, the operator $R_{2}(\alpha+1)$ is bounded from $L^{2}(\mathbb{R})$ into $L^{2}(\mathbb{R})$.

Hence replacing $P_{2}(\alpha+1)$ into $B_{2,2}$ and $B_{2,3}$ lead us to, in first place that

$$
\begin{aligned}
B_{2,2}(t)= & \left(\frac{\alpha+1}{4}\right) \int_{\mathbb{R}}\left(D_{x}^{2+\alpha} u\right)^{2}\left(\chi_{\epsilon, b}^{2}\right)^{\prime} \mathrm{d} x-c_{3}\left(\frac{\alpha+1}{16}\right) \int_{\mathbb{R}}\left(D_{x}^{1+\alpha} u\right)^{2}\left(\chi_{\epsilon, b}^{2}\right)^{(3)} \mathrm{d} x \\
& +c_{5}\left(\frac{\alpha+1}{64}\right) \int_{\mathbb{R}}\left(D_{x}^{\alpha} u\right)^{2}\left(\chi_{\epsilon, b}^{2}\right)^{(5)} \mathrm{d} x \\
= & B_{2,2,1}(t)+B_{2,2,2}(t)+B_{2,2,3}(t),
\end{aligned}
$$

and in second place

$$
\begin{aligned}
B_{2,3}(t)= & \left(\frac{\alpha+1}{4}\right) \int_{\mathbb{R}}\left(\mathcal{H} D_{x}^{2+\alpha} u\right)^{2}\left(\chi_{\epsilon, b}^{2}\right)^{\prime} \mathrm{d} x-c_{3}\left(\frac{\alpha+1}{16}\right) \int_{\mathbb{R}}\left(\mathcal{H} D_{x}^{1+\alpha} u\right)^{2}\left(\chi_{\epsilon, b}^{2}\right)^{(3)} \mathrm{d} x \\
& +c_{5}\left(\frac{\alpha+1}{64}\right) \int_{\mathbb{R}}\left(\mathcal{H} D_{x}^{\alpha} u\right)^{2}\left(\chi_{\epsilon, b}^{2}\right)^{(5)} \mathrm{d} x \\
= & B_{2,3,1}(t)+B_{2,3,2}(t)+B_{2,3,3}(t) .
\end{aligned}
$$

The terms $B_{2,2,1}$ and $B_{2,3,1}$ are positives and give us the smoothing effect. We estimate next $B_{2,2,2}$ and $B_{2,3,2}$. To avoid repetitions, we only show the procedures how to bound $B_{2,3,2}$. A similar analysis is applied to $B_{2,2,2}$.

Before carry on, we shall remember that for any $\epsilon>0$ and $b \geqslant 5 \epsilon$ the function $\varphi_{\epsilon, b}=$ $\sqrt{\chi_{\epsilon, b}^{\prime}}$ is smooth.

Besides,

$$
\varphi_{\epsilon / 3, b+\epsilon} D_{x}^{1+\alpha} u=D_{x}^{1+\alpha}\left(u \varphi_{\epsilon / 3, b+\epsilon}\right)-\left[D_{x}^{1+\alpha} ; \varphi_{\epsilon / 3, b+\epsilon}\right] u
$$


then a combination of Lemma 3.3 and interpolation produce

$$
\begin{aligned}
\left\|\varphi_{\epsilon / 3, b+\epsilon} D_{x}^{1+\alpha} u\right\|_{2} & \lesssim\left\|D_{x}^{1+\alpha}\left(u \varphi_{\epsilon / 3, b+\epsilon}\right)\right\|_{2}+\left\|\varphi_{\epsilon / 3, b+\epsilon}\right\|_{l, 2}\|u\|_{\alpha, 2} \\
& \lesssim\left\|\partial_{x}^{2}\left(u \varphi_{\epsilon / 3, b+\epsilon}\right)\right\|_{2}+\left\|\varphi_{\epsilon / 3, b+\epsilon}\right\|_{l, 2}\|u\|_{\alpha, 2} \\
& \lesssim\left\|\partial_{x}^{2} u \varphi_{\epsilon / 3, b+\epsilon}\right\|_{2}+\|u\|_{S_{\alpha, 2}}+\left\|\varphi_{\epsilon / 3, b+\epsilon}\right\|_{l, 2}\|u\|_{\alpha, 2} .
\end{aligned}
$$

Thus,

$$
\begin{aligned}
\int_{0}^{T}\left|B_{2,2,2}(t)\right| \mathrm{d} t & =c\left\|\varphi_{\epsilon / 3, b+\epsilon} D_{x}^{1+\alpha} u\right\|_{L_{T}^{2} L_{x}^{2}}^{2} \\
& \lesssim\left\|\partial_{x}^{2} u \varphi_{\epsilon / 3, b+\epsilon}\right\|_{L_{T}^{2} L_{x}^{2}}^{2}+\|u\|_{L_{T}^{\infty} H_{x}^{s \alpha}}^{2} \\
& \leqslant c\left(\left\|u_{0}\right\|_{S_{\alpha}, 2} ; \epsilon ; v ; T\right),
\end{aligned}
$$

where the last inequality is a consequence of the local theory, interpolation and (5.2).

Similarly,

$$
\begin{aligned}
\int_{0}^{T}\left|B_{2,3,2}(t)\right| \mathrm{d} t & \lesssim \int_{0}^{T} \int_{\mathbb{R}}\left(\mathcal{H} D_{x}^{1+\alpha} u\right)^{2} \chi_{\epsilon / 3, b+\epsilon}^{\prime} \mathrm{d} x \mathrm{~d} t \\
& \leqslant c\left(\left\|u_{0}\right\|_{\mathcal{S}_{\alpha}, 2} ; \epsilon ; v ; T\right) .
\end{aligned}
$$

After integrate in time

$$
\int_{0}^{T}\left|B_{2,2+l, 3}(t)\right| \mathrm{d} t<\infty \quad \text { for } \quad l \in\{0,1\}
$$

$\S .3$ Finally, we deal with the term $B_{3}$, which corresponds to the nonlinear part of the equation in (1.1).

First, we decompose the nonlinearity as follows

$$
\begin{aligned}
\partial_{x}^{2} D_{x}^{\frac{\alpha}{2}}\left(u \partial_{x} u\right) \chi_{\epsilon, b}= & -D_{x}^{2+\frac{\alpha}{2}}\left(u \partial_{x} u\right) \chi_{\epsilon, b} \\
= & \frac{1}{2}\left[D_{x}^{2+\frac{\alpha}{2}} ; \chi_{\epsilon, b}\right] \partial_{x}\left(\left(u \chi_{\epsilon, b}\right)^{2}+\left(u \widetilde{\phi_{\epsilon, b}}\right)^{2}+u^{2} \psi_{\epsilon}\right) \\
& -\left[D_{x}^{2+\frac{\alpha}{2}} ; u \chi_{\epsilon, b}\right] \partial_{x}\left(u \chi_{\epsilon, b}+u \phi_{\epsilon, b}+u \psi_{\epsilon}\right)+u \chi_{\epsilon, b} \partial_{x}^{2} D_{x}^{\frac{\alpha}{2}} \partial_{x} u \\
= & \widetilde{B_{3,1}}(t)+\widetilde{B_{3,2}}(t)+\widetilde{B_{3,3}}(t)+\widetilde{B_{3,4}}(t)+\widetilde{B_{3,5}}(t)+\widetilde{B_{3,6}}(t)+\widetilde{B_{3,7}}(t) .
\end{aligned}
$$

To estimate $B_{3}$ is sufficient the $L^{2}(\mathbb{R})-$ norm of the terms $\widetilde{B_{3, l}}$ for $l=1,2, \ldots, 7$.

Combining Lemma 3.1 and Lemma 3.3 it is obtained

$$
\begin{aligned}
\left\|\widetilde{B_{3,1}}\right\|_{2} & \lesssim\left\|\partial_{x} \chi_{\epsilon, b}\right\|_{l, 2}\left\|\left(u \chi_{\epsilon, b}\right)^{2}\right\|_{2+\alpha / 2,2} \\
& \lesssim\left\|\left(u \chi_{\epsilon, b}\right)^{2}\right\|_{2}+\left\|D_{x}^{2+\frac{\alpha}{2}}\left(\left(u \chi_{\epsilon, b}\right)^{2}\right)\right\|_{2} \\
& \lesssim\|u\|_{\infty}\left(\left\|u_{0}\right\|_{2}+\left\|D_{x}^{2+\frac{\alpha}{2}}\left(u \chi_{\epsilon, b}\right)\right\|_{2}\right)
\end{aligned}
$$

and

$$
\begin{aligned}
\left\|\widetilde{B_{3,2}}\right\|_{2} & \lesssim\left\|\partial_{x} \chi_{\epsilon, b}\right\|_{l, 2}\left\|\left(u \phi_{\epsilon, b}\right)^{2}\right\|_{2+\alpha / 2,2} \\
& \lesssim\|u\|_{\infty}\left(\left\|u_{0}\right\|_{2}+\left\|D_{x}^{2+\frac{\alpha}{2}}\left(u \phi_{\epsilon, b}\right)\right\|_{2}\right) .
\end{aligned}
$$


Since the weighted functions $\chi_{\epsilon, b}$ and $\psi_{\epsilon}$ satisfy hypothesis of Lemma 3.5, then

$$
\left\|\widetilde{B_{3,3}}\right\|_{2}=\left\|\chi_{\epsilon, b} D_{x}^{2+\frac{\alpha}{2}}\left(u^{2} \psi_{\epsilon}\right)\right\|_{2} \lesssim\|u\|_{\infty}\left\|u_{0}\right\|_{2},
$$

and

$$
\left\|\widetilde{B_{3,6}}\right\|_{2}=\left\|u \chi_{\epsilon, b} D_{x}^{2+\frac{\alpha}{2}}\left(u \psi_{\epsilon}\right)\right\|_{2} \lesssim\left\|u_{0}\right\|_{2}\|u\|_{\infty} .
$$

To handle the terms $\widetilde{B_{3,4}}$ and $\widetilde{B_{3,1}}$, we use the commutator estimate (3.9) to yield

$$
\left\|\widetilde{B_{3,4}}\right\|_{2} \lesssim\left\|D_{x}^{2+\frac{\alpha}{2}}\left(u \chi_{\epsilon, b}\right)\right\|_{2}\left\|\partial_{x}\left(u \chi_{\epsilon, b}\right)\right\|_{\infty}
$$

and

$$
\left\|\widetilde{B_{3,5}}\right\|_{2} \lesssim\left\|D_{x}^{2+\frac{\alpha}{2}}\left(u \phi_{\epsilon, b}\right)\right\|_{2}\left\|\partial_{x}\left(u \chi_{\epsilon, b}\right)\right\|_{\infty}+\left\|D_{x}^{2+\frac{\alpha}{2}}\left(u \chi_{\epsilon, b}\right)\right\|_{2}\left\|\partial_{x}\left(u \phi_{\epsilon, b}\right)\right\|_{\infty} .
$$

The nonlocal character of the operator $D_{x}^{s}$, for $s \notin 2 \mathbb{N}$, implies that several terms above shall be estimated. First, an immediate application of Theorem 3.4 yield

$$
\begin{aligned}
\left\|D_{x}^{2+\frac{\alpha}{2}}\left(u \phi_{\epsilon, b}\right)\right\|_{2} \lesssim & \left\|D_{x}^{2+\frac{\alpha}{2}} \phi_{\epsilon, b}\right\|_{\mathrm{BMO}}\|u\|_{2}+\left\|\phi_{\epsilon, b} D_{x}^{2+\frac{\alpha}{2}} u\right\|_{2}+\left\|\partial_{x} \phi_{\epsilon, b} \mathcal{H} D_{x}^{1+\frac{\alpha}{2}} u\right\|_{2} \\
& +\left\|\partial_{x}^{2} \phi_{\epsilon, b} D_{x}^{\frac{\alpha}{2}} u\right\|_{2} \\
\lesssim & \left\|D_{x}^{\frac{\alpha}{2}} \partial_{x}^{2} \phi_{\epsilon, b}\right\|_{\infty}\left\|u_{0}\right\|_{2}+\left\|\chi_{\epsilon / 8, b+\epsilon / 4}^{\prime} D_{x}^{2+\frac{\alpha}{2}} u\right\|_{2}+\|u\|_{s_{\alpha}, 2} \\
\lesssim & \lesssim \chi_{\epsilon / 8, b+\epsilon / 4}^{\prime} D_{x}^{2+\frac{\alpha}{2}} u\left\|_{2}+\right\| u \|_{s_{\alpha}, 2} .
\end{aligned}
$$

Notice that the second term on the right hand side is bounded by local theory. By using the weighted functions properties combined with (5.8) it follows

$$
\begin{aligned}
\int_{0}^{T}\left\|D_{x}^{2+\frac{\alpha}{2}} u \chi_{\epsilon / 8, b+\epsilon / 4}\right\|_{2} \mathrm{~d} t & \leqslant T^{1 / 2}\left\|D_{x}^{2+\frac{\alpha}{2}} u \chi_{\epsilon / 8, b+\epsilon / 4}^{\prime}\right\|_{L_{T}^{2} L_{x}^{2}} \\
& \lesssim T^{1 / 2}\left\|D_{x}^{2+\frac{\alpha}{2}} u \eta_{\epsilon / 24, b+7 \epsilon / 24}\right\|_{L_{T}^{2} L_{x}^{2}} \\
& \lesssim\left(c_{2,1}^{*}\right)^{1 / 2} .
\end{aligned}
$$

Similarly

$$
\left\|D_{x}^{2+\frac{\alpha}{2}}\left(u \widetilde{\phi_{\epsilon, b}}\right)\right\|_{L_{T}^{1} L_{x}^{2}} \lesssim\left(c_{2,1}^{*}\right)^{1 / 2}
$$

Finally, to estimate $\left\|D_{x}^{2+\frac{\alpha}{2}}\left(u \chi_{\epsilon, b}\right)\right\|_{2}$, we write

$$
D_{x}^{2+\frac{\alpha}{2}}\left(u \chi_{\epsilon, b}\right)=-\chi_{\epsilon, b} \partial_{x}^{2} D_{x}^{\frac{\alpha}{2}} u+\left[D_{x}^{2+\frac{\alpha}{2}} ; \chi_{\epsilon, b}\right]\left(u \chi_{\epsilon, b}+u \phi_{\epsilon, b}+u \psi_{\epsilon}\right) .
$$

Thus, a combination of Lemma 3.3 and interpolation lead us to

$$
\left\|D_{x}^{2+\frac{\alpha}{2}}\left(u \chi_{\epsilon, b}\right)\right\|_{2} \lesssim\left\|\chi_{\epsilon, b} \partial_{x}^{2} D_{x}^{\frac{\alpha}{2}} u\right\|_{2}+\|u\|_{S_{\alpha}, 2} .
$$

Notice that the first term on the right hand side is the quantity to be estimated by Gronwall's inequality.

Concerning to $B_{3,7}$ we obtain after apply integration by parts

$$
\begin{aligned}
B_{3,7}(t) & =-\frac{1}{2} \int_{\mathbb{R}} \partial_{x} u \chi_{\epsilon, b}^{2}\left(\partial_{x}^{2} D_{x}^{\frac{\alpha}{2}} u\right)^{2} \mathrm{~d} x-\frac{1}{2} \int_{\mathbb{R}} u\left(\chi_{\epsilon, b}^{2}\right)^{\prime}\left(\partial_{x}^{2} D_{x}^{\frac{\alpha}{2}} u\right)^{2} \mathrm{~d} x \\
& =B_{3,7,1}(t)+B_{3,7,2}(t) .
\end{aligned}
$$


On one hand, we have

$$
\left|B_{3,7,1}(t)\right| \lesssim\left\|\partial_{x} u(t)\right\|_{\infty} \int_{\mathbb{R}}\left(\partial_{x}^{2} D_{x}^{\frac{\alpha}{2}} u\right)^{2} \chi_{\epsilon, b}^{2} \mathrm{~d} x,
$$

the integral expression on the right hand side will be estimate by Gronwall's inequality and by Theorem 1.2 we have $\partial_{x} u \in L_{T}^{1} L_{x}^{\infty}$.

On the other hand, by Sobolev's embedding it follows that

$$
\begin{aligned}
\left|B_{3,7,2}(t)\right| & \lesssim\|u(t)\|_{\infty} \int_{\mathbb{R}}\left(\chi_{\epsilon, b}^{2}\right)^{\prime}\left(D_{x}^{2+\frac{\alpha}{2}} u\right)^{2} \mathrm{~d} x \\
& \lesssim \sup _{0 \leqslant t \leqslant T}\|u(t)\|_{s_{\alpha}, 2} \int_{\mathbb{R}}\left(\chi_{\epsilon, b}^{2}\right)^{\prime}\left(\partial_{x}^{2} D_{x}^{\frac{\alpha}{2}} u\right)^{2} \mathrm{~d} x .
\end{aligned}
$$

Integrating in time yields

$$
\int_{0}^{T}\left|B_{3,7,2}(t)\right| \mathrm{d} t \lesssim \sup _{0 \leqslant t \leqslant T}\|u(t)\|_{s_{\alpha}, 2} \int_{0}^{T} \int_{\mathbb{R}}\left(\chi_{\epsilon, b}^{2}\right)^{\prime}\left(\partial_{x}^{2} D_{x}^{\frac{\alpha}{2}} u\right)^{2} \mathrm{~d} x \mathrm{~d} t
$$

where the integral expression corresponds to the $B_{1}$ term already estimated in (5.9).

Gathering the estimates corresponding to $B_{1}, B_{2}$ and $B_{3}$ combined with Gronwall's inequality and integration in time yields that for any $\epsilon>0, b \geqslant 5 \epsilon$ and $v \geqslant 0$

$$
\sup _{0 \leqslant t \leqslant T}\left\|\partial_{x}^{2} D_{x}^{\frac{\alpha}{2}} u \chi_{\epsilon, b}(\cdot+v t)\right\|_{2}^{2}+\left\|D_{x}^{2+\alpha} u \eta_{\epsilon, b}\right\|_{L_{T}^{2} L_{x}^{2}}^{2}+\left\|\mathcal{H} D_{x}^{2+\alpha} u \eta_{\epsilon, b}\right\|_{L_{T}^{2} L_{x}^{2}}^{2} \leqslant c_{2,2}^{*},
$$

where $c_{2,2}^{*}=c_{2,2}^{*}\left(\alpha ; \epsilon ; T ; v ;\left\|u_{0}\right\|_{s_{\alpha}, 2} ;\left\|D_{x}^{\frac{\alpha}{2}} \partial_{x}^{2} u_{0} \chi_{\epsilon, b}\right\|_{2}\right)>0$.

At this point, we shall determine the number of steps in the second inductive process that allow us to reach the next integer. For this reason we will consider the following cases:

(a) If $\frac{2}{2 k+1} \leqslant \alpha<\frac{1}{k}$ for some positive integer $k$, then are required $2 k+1$ steps in the second inductive process.

(b) Instead, if $\frac{1}{k+1} \leqslant \alpha<\frac{2}{2 k+1}$ for some positive integer $k$, then are required $2 k+2$ steps in the second inductive process.

A detailed description of the number of steps can be obtained directly from (a)-(b).More precisely, there are required $\left\lceil\frac{2}{\alpha}\right\rceil$ steps in both cases, where $\lceil\cdot\rceil$ denotes the ceiling function. The different considerations are made to differentiate when the number of steps are even or odd. A graphical description of the process described here is presented below.

Henceforth, for comfort in the notation we will consider $\alpha$ satisfying the condition (a).

As part of the second inductive process we shall assume that

$$
\sup _{0 \leqslant t \leqslant T}\left\|\partial_{x}^{2} D_{x}^{\frac{\alpha j}{2}} u \chi_{\epsilon, b}(\cdot+v t)\right\|_{2}^{2}+\left\|D_{x}^{2+\alpha\left(\frac{j+1}{2}\right)} u \eta_{\epsilon, b}\right\|_{L_{T}^{2} L_{x}^{2}}^{2}+\left\|\mathcal{H} D_{x}^{2+\alpha\left(\frac{j+1}{2}\right)} u \eta_{\epsilon, b}\right\|_{L_{T}^{2} L_{x}^{2}}^{2} \leqslant c_{2, j+1}^{*}
$$

for every $\epsilon>0, b \geqslant 5 \epsilon, v \geqslant 0$, and $j=0,1, \cdots, 2 k-1$.

In fact, the previous cases in the induction process are summarized in the following diagram: 


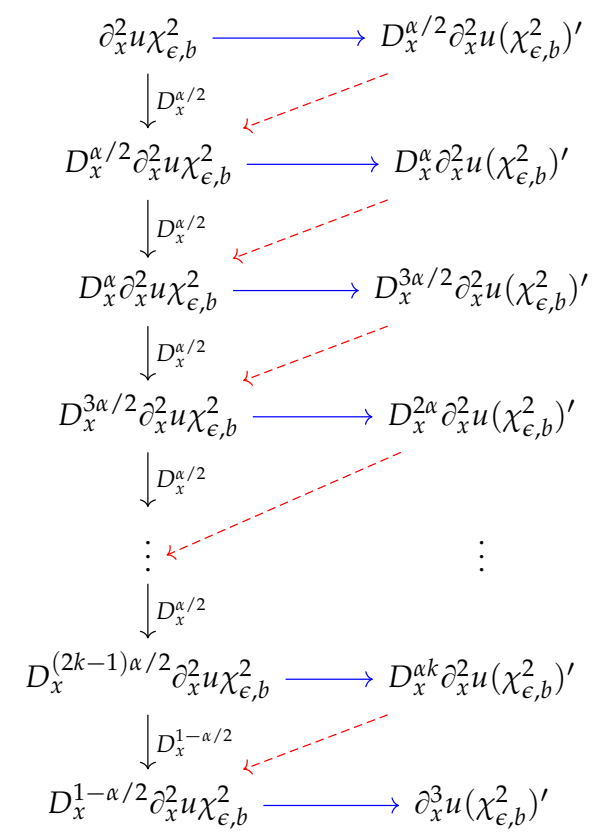

The last before the last case in the diagram is the one that we will carry out in the next step, we will present all the issues corresponding to this case.

STEP $2 k+1$ :

A standard argument lead us to the energy identity

$$
\begin{aligned}
& \frac{1}{2} \frac{\mathrm{d}}{\mathrm{d} t} \int_{\mathbb{R}}\left(\partial_{x}^{2} D_{x}^{1-\frac{\alpha}{2}} u\right)^{2} \chi_{\epsilon, b}^{2} \mathrm{~d} x-\underbrace{-\frac{v}{2} \int_{\mathbb{R}}\left(\partial_{x}^{2} D_{x}^{1-\frac{\alpha}{2}} u\right)^{2}\left(\chi_{\epsilon, b}^{2}\right)^{\prime} \mathrm{d} x}_{B_{1}(t)} \\
& \underbrace{-\int_{\mathbb{R}}\left(\partial_{x}^{2} D_{x}^{1-\frac{\alpha}{2}} D_{x}^{\alpha} \partial_{x} u\right) \partial_{x}^{2} D_{x}^{1-\frac{\alpha}{2}} u \chi_{\epsilon, b}^{2} \mathrm{~d} x}_{B_{2}(t)} \\
& +\underbrace{\underbrace{\int_{\mathbb{R}}\left(\partial_{x}^{2} D_{x}^{1-\frac{\alpha}{2}}\left(u \partial_{x} u\right)\right) \partial_{x}^{2} D_{x}^{1-\frac{\alpha}{2}} u \chi_{\epsilon, b}^{2} \mathrm{~d} x}_{\mathbb{R}}}_{B_{3}(t)}, \quad .
\end{aligned}
$$

Notice that estimating the term $\left\|B_{1}\right\|_{2}$ is straightforward in the previous cases, because it is a direct consequence from the former cases. However, this cannot be done in this step, instead a new approach is required.

Since the smoothing effect obtained in the case $j=2 k-1$ is $2+\alpha k$, then by hypothesis

$$
2+\alpha k \geqslant 3-\frac{\alpha}{2} \text { for } \alpha \in\left[\frac{2}{2 k+1}, \frac{1}{k}\right) .
$$

Therefore, the regularity obtained in (5.22) is enough to provide a bound for $\left\|B_{1}\right\|_{1}$. To do this, we first decompose the term $\partial_{x}^{2} D_{x}^{\alpha k} u$ as follows:

$$
\partial_{x}^{2} D_{x}^{\alpha k}\left(u \eta_{\epsilon, b}\right)=\eta_{\epsilon, b} \partial_{x}^{2} D_{x}^{\alpha k} u-\left[D_{x}^{2+\alpha k} ; \eta_{\epsilon, b}\right]\left(u \chi_{\epsilon, b}+u \phi_{\epsilon, b}+u \psi_{\epsilon}\right) .
$$


Then, by Lemma 3.3

$$
\begin{aligned}
\left\|\partial_{x}^{2} D_{x}^{\alpha k}\left(u \eta_{\epsilon, b}\right)\right\|_{2} \leqslant & \left\|\partial_{x}^{2} D_{x}^{\alpha k} u \eta_{\epsilon, b}\right\|_{2}+\left\|\left[D_{x}^{2+\alpha k} ; \eta_{\epsilon, b}\right]\left(u \chi_{\epsilon, b}+u \phi_{\epsilon, b}+u \psi_{\epsilon}\right)\right\|_{2} \\
\lesssim & \left\|\partial_{x}^{2} D_{x}^{\alpha k} u \eta_{\epsilon, b}\right\|_{2}+\left\|\eta_{\epsilon, b}\right\|_{l, 2}\left(\left\|u \chi_{\epsilon, b}\right\|_{1+\alpha k, 2}+\left\|u \phi_{\epsilon, b}\right\|_{1+\alpha k, 2}\right) \\
& +\left\|\eta_{\epsilon, b} D_{x}^{2+\alpha k}\left(u \psi_{\epsilon}\right)\right\|_{2} \\
= & I_{1}+I_{2}+I_{3}+I_{4} .
\end{aligned}
$$

Notice that after integrate in time, the inequality (5.22) implies that

$$
\left\|I_{1}\right\|_{L_{T}^{2}}=\left(\int_{0}^{T} \int_{\mathbb{R}}\left(D_{x}^{2+\alpha k} u\right)^{2} \eta_{\epsilon, b}^{2} \mathrm{~d} x \mathrm{~d} t\right)^{1 / 2} \lesssim\left(c_{2, j+1}^{*}\right)^{1 / 2} .
$$

The terms $I_{2}$ and $I_{3}$ can be bounded via Lemma 3.4 and Young's inequality

$$
\begin{aligned}
\left\|D_{x}^{1+\alpha k}\left(u \chi_{\epsilon, b}\right)\right\|_{2} & \lesssim\left\|\partial_{x}^{2}\left(u \chi_{\epsilon, b}\right)\right\|_{2}+\left\|u_{0}\right\|_{2} \\
& \lesssim\left\|\partial_{x}^{2} u \chi_{\epsilon, b}\right\|_{2}+\|u\|_{S_{\alpha, 2},}
\end{aligned}
$$

and

$$
\begin{aligned}
\left\|D_{x}^{1+\alpha k}\left(u \phi_{\epsilon, b}\right)\right\|_{2} & \lesssim\left\|\partial_{x}^{2}\left(u \phi_{\epsilon, b}\right)\right\|_{2}+\left\|u_{0}\right\|_{2} \\
& \lesssim\left\|\partial_{x}^{2} u \phi_{\epsilon, b}\right\|_{2}+\|u\|_{S_{\alpha}, 2} .
\end{aligned}
$$

Moreover,

$$
\left\|I_{2}\right\|_{L_{T}^{2}}=\left\|D_{x}^{1+\alpha k}\left(u \chi_{\epsilon, b}\right)\right\|_{L_{T}^{2} L_{x}^{2}} \lesssim \sup _{0 \leqslant t \leqslant T}\left\|\partial_{x}^{2} u \chi_{\epsilon, b}\right\|_{2}+\|u\|_{L_{T}^{\infty} H_{x}^{s \alpha}} .
$$

On the other hand, there exists $\tau>0$ such that

$$
\phi_{\epsilon, b}(x+v t) \lesssim \mathbb{1}_{[-\tau, \tau]}(x) \quad \text { for all } \quad(x, t) \in \mathbb{R} \times[0, T],
$$

Thus, in view of Remark 3.18

$$
\begin{aligned}
\left\|\partial_{x}^{2} u \phi_{\epsilon, b}\right\|_{L_{T}^{2} L_{x}^{2}} & \lesssim\left(\int_{0}^{T} \int_{\mathbb{R}}\left(\partial_{x}^{2} u\right)^{2} \phi_{\epsilon, b}^{2} \mathrm{~d} x \mathrm{~d} t\right)^{1 / 2} \\
& \lesssim\left(\int_{0}^{T} \int_{-\tau}^{\tau}\left(\partial_{x}^{2} u\right)^{2} \mathrm{~d} x \mathrm{~d} t\right)^{1 / 2} \\
& \leqslant c\left(\left\|u_{0}\right\|_{s_{\alpha}, 2} ; \tau\right) .
\end{aligned}
$$

Next, by Lemma 3.5, it follows that

$$
\left\|\eta_{\epsilon, b} D_{x}^{2+\alpha k}\left(u \psi_{\epsilon}\right)\right\|_{2} \lesssim\left\|u_{0}\right\|_{2}
$$

Gathering together the estimates above lead us to

$$
\left\|D_{x}^{2+\alpha k}\left(u \eta_{\epsilon, b}\right)\right\|_{L_{T}^{2} L_{x}^{2}}<\infty .
$$

This inequality combined with Lemma 3.4 implies that

$$
\left\|D_{x}^{3-\frac{\alpha}{2}}\left(u \eta_{\epsilon, b}\right)\right\|_{2} \lesssim\left\|D_{x}^{2+\alpha k}\left(u \eta_{\epsilon, b}\right)\right\|_{2}^{\frac{6-\alpha}{2(2+\alpha k)}}\left\|u \eta_{\epsilon, b}\right\|_{2}^{\frac{\alpha(1+2 k)-2}{2(2+\alpha k)}} .
$$


Since we need to estimate $\partial_{x}^{2} D_{x}^{1-\frac{\alpha}{2}} u \eta_{\epsilon, b}$, a decomposition as that in (5.25) is sufficient to our proposes, i.e.

$$
\partial_{x}^{2} D_{x}^{1-\frac{\alpha}{2}} u \eta_{\epsilon, b}=\partial_{x}^{2} D_{x}^{1-\frac{\alpha}{2}}\left(u \eta_{\epsilon, b}\right)-\left[\partial_{x}^{2} D_{x}^{1-\frac{\alpha}{2}} ; \eta_{\epsilon, b}\right]\left(u \chi_{\epsilon, b}+u \phi_{\epsilon, b}+u \psi_{\epsilon}\right) .
$$

For the sake of brevity, we omit the computations behind these commutators estimates. Nevertheless, similar arguments as those used in (5.26)-(5.27) lead to

$$
\left\|\partial_{x}^{2} D_{x}^{1-\frac{\alpha}{2}} u \eta_{\epsilon, b}\right\|_{L_{T}^{2} L_{x}^{2}}<\infty .
$$

The estimates above are in fact a way to bound the term $\left\|B_{1}\right\|_{1}$ in (5.23).

More precisely,

$$
\int_{0}^{T}\left|B_{1}(t)\right| \mathrm{d} t=v\left\|\partial_{x}^{2} D_{x}^{1-\frac{\alpha}{2}} u \eta_{\epsilon, b}\right\|_{L_{T}^{2} L_{x}^{2}}^{2}<\infty
$$

$\S .2$ A previous argument (see step 1) implies that

$$
\begin{aligned}
B_{2}(t)= & \frac{1}{2} \int_{\mathbb{R}} D_{x}^{3-\frac{\alpha}{2}} u R_{n}(\alpha+1) D_{x}^{3-\frac{\alpha}{2}} u \mathrm{~d} x+\frac{1}{4} \int_{\mathbb{R}} D_{x}^{3-\frac{\alpha}{2}} u P_{n}(\alpha+1) D_{x}^{3-\frac{\alpha}{2}} u \mathrm{~d} x \\
& -\frac{1}{4} \int_{\mathbb{R}} D_{x}^{3-\frac{\alpha}{2}} u \mathcal{H} P_{n}(\alpha+1) \mathcal{H} D_{x}^{3-\frac{\alpha}{2}} u \mathrm{~d} x \\
= & B_{2,1}(t)+B_{2,2}(t)+B_{2,3}(t) .
\end{aligned}
$$

A similar argument to the used in step 1 allow us to fix $n=2$ above.

According to Proposition 3.1, the remainder term $R_{2}(\alpha+1)$ is a bounded operator in $L^{2}(\mathbb{R})$. Therefore

$$
B_{2,1}(t)=\frac{1}{2} \int_{\mathbb{R}} u D_{x}^{3-\frac{\alpha}{2}} R_{2}(\alpha+1) D_{x}^{3-\frac{\alpha}{2}} u \mathrm{~d} x,
$$

and by Hölder's inequality

$$
\left|B_{2,1}(t)\right| \lesssim\left\|u_{0}\right\|_{2}\left\|D_{x}^{3-\frac{\alpha}{2}} R_{2}(\alpha+1) D_{x}^{3-\frac{\alpha}{2}} u\right\|_{2} \lesssim\left\|u_{0}\right\|_{2}^{2} \| \widehat{D_{x}^{7}\left(\chi_{\epsilon, b}^{2}\right) \|_{1}} .
$$

Thus,

$$
\int_{0}^{T}\left|B_{2,1}(t)\right| \mathrm{d} t \lesssim T\left\|u_{0}\right\|_{2}^{2}\left\|\widehat{D_{x}^{7}\left(\chi_{\epsilon, b}^{2}\right)}\right\|_{1} .
$$

If we decompose the terms in (5.29) it follows that

$$
\begin{aligned}
B_{2,2}(t)= & \left(\frac{\alpha+1}{4}\right) \int_{\mathbb{R}}\left(\mathcal{H} \partial_{x}^{3} u\right)^{2}\left(\chi_{\epsilon, b}^{2}\right)^{\prime} \mathrm{d} x-c_{3}\left(\frac{\alpha+1}{16}\right) \int_{\mathbb{R}}\left(\partial_{x}^{2} u\right)^{2}\left(\chi_{\epsilon, b}^{2}\right)^{(3)} \mathrm{d} x \\
& +c_{5}\left(\frac{\alpha+1}{64}\right) \int_{\mathbb{R}}\left(\mathcal{H} \partial_{x} u\right)^{2}\left(\chi_{\epsilon, b}^{2}\right)^{(5)} \mathrm{d} x \\
= & B_{2,2,1}(t)+B_{2,2,2}(t)+B_{2,2,3}(t),
\end{aligned}
$$

and

$$
\begin{aligned}
B_{2,3}(t)= & \left(\frac{\alpha+1}{4}\right) \int_{\mathbb{R}}\left(\partial_{x}^{3} u\right)^{2}\left(\chi_{\epsilon, b}^{2}\right)^{\prime} \mathrm{d} x-c_{3}\left(\frac{\alpha+1}{16}\right) \int_{\mathbb{R}}\left(\mathcal{H} \partial_{x}^{2} u\right)^{2}\left(\chi_{\epsilon, b}^{2}\right)^{(3)} \mathrm{d} x \\
& +c_{5}\left(\frac{\alpha+1}{64}\right) \int_{\mathbb{R}}\left(\partial_{x} u\right)^{2}\left(\chi_{\epsilon, b}^{2}\right)^{(5)} \mathrm{d} x \\
= & B_{2,3,1}(t)+B_{2,3,2}(t)+B_{2,3,3}(t) .
\end{aligned}
$$

As before $B_{2,2,1}$ and $B_{2,3,1}$ provide the smoothing effect after integration in time. 
The terms $B_{2,2,2}$ and $B_{2,3,2}$ are easily handled by using Proposition 3.18 and the arguments used in (5.1)-(5.2). Meanwhile, the low regularity in the terms $B_{2,2,3}$ and $B_{2,3,3}$ is handled by using local theory.

$\S .3$ To finish this step, we turn our attention to the term involving the nonlinear part of the equation

$$
\begin{aligned}
\partial_{x}^{2} D_{x}^{1-\frac{\alpha}{2}}\left(u \partial_{x} u\right) \chi_{\epsilon, b}= & -D_{x}^{3-\frac{\alpha}{2}}\left(u \partial_{x} u\right) \chi_{\epsilon, b} \\
= & \frac{1}{2}\left[D_{x}^{3-\frac{\alpha}{2}} ; \chi_{\epsilon, b}\right] \partial_{x}\left(\left(u \chi_{\epsilon, b}\right)^{2}+\left(u \widetilde{\phi_{\epsilon, b}}\right)^{2}+u^{2} \psi_{\epsilon}\right) \\
& -\left[D_{x}^{3-\frac{\alpha}{2}} ; u \chi_{\epsilon, b}\right] \partial_{x}\left(u \chi_{\epsilon, b}+u \phi_{\epsilon, b}+u \psi_{\epsilon}\right)+u \chi_{\epsilon, b} \partial_{x}^{2} D_{x}^{1-\frac{\alpha}{2}} \partial_{x} u \\
= & \widetilde{B_{3,1}}(t)+\widetilde{B_{3,2}}(t)+\widetilde{B_{3,3}}(t)+\widetilde{B_{3,4}}(t)+\widetilde{B_{3,5}}(t)+\widetilde{B_{3,6}}(t)+\widetilde{B_{3,7}}(t) .
\end{aligned}
$$

In the decomposition above is sufficient to estimate the $L_{x}^{2}-$ norm of the terms $\widetilde{B_{3, l}}$ for $l=1,2, \cdots, 6$.

Combining (3.3) and Lemma 3.3 it follows that

$$
\begin{aligned}
\left\|\widetilde{B_{3,1}}\right\|_{2} & \lesssim\left\|\chi_{\epsilon, b}^{\prime}\right\|_{l, 2}\left\|\left(u \chi_{\epsilon, b}\right)^{2}\right\|_{3-\frac{\alpha}{2}, 2} \\
& \lesssim\left\|u^{2}\right\|_{2}+\left\|D_{x}^{3-\frac{\alpha}{2}}\left(\left(u \chi_{\epsilon, b}\right)^{2}\right)\right\|_{2} \\
& \lesssim\|u\|_{\infty}\left(\left\|u_{0}\right\|_{2}+\left\|D_{x}^{3-\frac{\alpha}{2}}\left(u \chi_{\epsilon, b}\right)\right\|_{2}\right)
\end{aligned}
$$

and

$$
\left\|\widetilde{B_{3,2}}\right\|_{2} \lesssim\|u\|_{\infty}\left(\left\|u_{0}\right\|_{2}+\left\|D_{x}^{3-\frac{\alpha}{2}}\left(u \phi_{\epsilon, b}\right)\right\|_{2}\right)
$$

Since $\chi_{\epsilon, b}$ and $\psi_{\epsilon}$ satisfy

$$
\operatorname{dist}\left(\operatorname{supp}\left(\chi_{\epsilon, b}\right), \operatorname{supp}\left(\psi_{\epsilon}\right)\right) \geqslant \frac{\epsilon}{2}>0,
$$

then by Lemma 3.5, it follows that

$$
\left\|\widetilde{B_{3,3}}\right\|_{2}=\left\|\chi_{\epsilon, b} D_{x}^{3-\frac{\alpha}{2}}\left(u^{2} \psi_{\epsilon}\right)\right\|_{2} \lesssim\|u\|_{\infty}\left\|u_{0}\right\|_{2}
$$

and

$$
\left\|\widetilde{B_{3,6}}\right\|_{2}=\left\|u \chi_{\epsilon, b} D_{x}^{3-\frac{\alpha}{2}}\left(u \psi_{\epsilon}\right)\right\|_{2} \lesssim\left\|u_{0}\right\|_{2}\|u\|_{\infty} .
$$

Instead, the terms $\widetilde{B_{3,4}}$ and $\widetilde{B_{3,1}}$ are handled by using (3.9)

$$
\left\|\widetilde{B_{3,4}}\right\|_{2} \lesssim\left\|D_{x}^{3-\frac{\alpha}{2}}\left(u \chi_{\epsilon, b}\right)\right\|_{2}\left\|\partial_{x}\left(u \chi_{\epsilon, b}\right)\right\|_{\infty}
$$

and

$$
\left\|\widetilde{B_{3,5}}\right\|_{2} \lesssim\left\|D_{x}^{3-\frac{\alpha}{2}}\left(u \phi_{\epsilon, b}\right)\right\|_{2}\left\|\partial_{x}\left(u \chi_{\epsilon, b}\right)\right\|_{\infty}+\left\|D_{x}^{3-\frac{\alpha}{2}}\left(u \chi_{\epsilon, b}\right)\right\|_{2}\left\|\partial_{x}\left(u \phi_{\epsilon, b}\right)\right\|_{\infty} .
$$

Notice that

$$
\partial_{x}^{2} D_{x}^{1-\frac{\alpha}{2}}\left(u \chi_{\epsilon, b}\right)=\chi_{\epsilon, b} \partial_{x}^{2} D_{x}^{1-\frac{\alpha}{2}} u-\left[D_{x}^{3-\frac{\alpha}{2}} ; \chi_{\epsilon, b}\right]\left(u \chi_{\epsilon, b}+u \phi_{\epsilon, b}+u \psi_{\epsilon}\right),
$$

so that a combination of interpolation, local theory and Lemma 3.3 yield

$$
\left\|\partial_{x}^{2} D_{x}^{1-\frac{\alpha}{2}}\left(u \chi_{\epsilon, b}\right)\right\|_{2} \lesssim\left\|\chi_{\epsilon, b} \partial_{x}^{2} D_{x}^{1-\frac{\alpha}{2}} u\right\|_{2}+\left\|\partial_{x}^{2} u \chi_{\epsilon, b}\right\|_{2}+\left\|\partial_{x}^{2} u \phi_{\epsilon, b}\right\|_{2}+\|u\|_{\mathcal{S}_{\alpha}, 2} .
$$


An immediate application of Theorem 3.4 implies that

$$
\begin{aligned}
& \left\|D_{x}^{2+\alpha k}\left(u \phi_{\epsilon, b}\right)\right\|_{2} \\
& \lesssim\left\|D_{x}^{2+\alpha k} \phi_{\epsilon, b}\right\|_{\mathrm{BMO}}\|u\|_{2}+\left\|\phi_{\epsilon, b} D_{x}^{2+\alpha k} u\right\|_{2}+\left\|\partial_{x} \phi_{\epsilon, b} \mathcal{H} D_{x}^{1+\alpha k} u\right\|_{2}+\left\|\partial_{x}^{2} \phi_{\epsilon, b} D_{x}^{\alpha k} u\right\|_{2} \\
& \lesssim\left\|u_{0}\right\|_{2}+\left\|\chi_{\epsilon / 8, b+\epsilon / 4}^{\prime} D_{x}^{2+\alpha k} u\right\|_{2}+\left\|\chi_{\epsilon / 8, b+\epsilon / 4}^{\prime} \mathcal{H} D_{x}^{1+\alpha k} u\right\|_{2}+\left\|\chi_{\epsilon / 8, b+\epsilon / 4}^{\prime} D_{x}^{\alpha k} u\right\|_{2} \\
& =A_{1}+A_{2}+A_{3}+A_{4} .
\end{aligned}
$$

The first and fourth term on the right hand side above are bounded after integrate in time. The second and third term require extra arguments to control them after integrate in time.

Since $\alpha$ satisfies

$$
\frac{2}{2 k+1} \leqslant \alpha<\frac{1}{k} \quad \text { for } \quad k \in \mathbb{Z}^{+},
$$

it is clear that $\alpha k$ is positive and strictly less than one for all positive integer $k$, then is straightforward to see that $\left\|A_{4}\right\|_{1}<\infty$.

Interpolation combined with Calderon's commutator estimate (3.2) implies that

$$
\begin{aligned}
A_{3} & =\left\|\partial_{x} \phi_{\epsilon, b} \mathcal{H} D_{x}^{1+\alpha k} u\right\|_{2} \\
& =\left\|\mathcal{H}\left(\partial_{x} \phi_{\epsilon, b} D_{x}^{1+\alpha k} u\right)-\left[\mathcal{H} ; \partial_{x} \phi_{\epsilon, b}\right] D_{x}^{1+\alpha k} u\right\|_{2} \\
& \lesssim\left\|\partial_{x} \phi_{\epsilon, b} D_{x}^{1+\alpha k} u\right\|_{2}+\|u\|_{S_{\alpha}, 2},
\end{aligned}
$$

and

$$
\begin{aligned}
\left\|\partial_{x} \phi_{\epsilon, b} D_{x}^{1+\alpha k} u\right\|_{2} & \lesssim\left\|\partial_{x}^{2}\left(u \partial_{x} \phi_{\epsilon, b}\right)\right\|_{2}+\|u\|_{s_{\alpha, 2}} \\
& \lesssim\left\|\partial_{x}^{2} u \chi_{\epsilon / 8, b+\epsilon / 4}^{\prime}\right\|_{2}+\|u\|_{s_{\alpha, 2}} .
\end{aligned}
$$

The last inequality above is obtained combining interpolation and the properties of the weighted functions.

Hence,

$$
\left\|A_{3}\right\|_{1} \lesssim c\left(\left\|u_{0}\right\|_{s_{\alpha}, 2} ; \epsilon ; T ; v\right)+\|u\|_{L_{T}^{\infty} H_{x}^{s \alpha}} .
$$

Next, the properties of weighted functions and (5.22) imply

$$
\left\|A_{2}\right\|_{1} \lesssim T^{1 / 2}\left(c_{2,2 k}^{*}\right)^{1 / 2} .
$$

Applying similar arguments allow us to estimate $\left\|D_{x}^{2+\alpha k}\left(u \widetilde{\phi_{\epsilon, b}}\right)\right\|_{2}$.

Finally, integration by parts give us

$$
\begin{aligned}
B_{3,7}(t) & =-\frac{1}{2} \int_{\mathbb{R}} \partial_{x} u \chi_{\epsilon, b}^{2}\left(\partial_{x}^{2} D_{x}^{1-\frac{\alpha}{2}} u\right)^{2} \mathrm{~d} x-\frac{1}{2} \int_{\mathbb{R}} u\left(\chi_{\epsilon, b}^{2}\right)^{\prime}\left(\partial_{x}^{2} D_{x}^{1-\frac{\alpha}{2}} u\right)^{2} \mathrm{~d} x \\
& =B_{3,7,1}(t)+B_{3,7,2}(t) .
\end{aligned}
$$

Observe that

$$
\left|B_{3,7,1}(t)\right| \lesssim\left\|\partial_{x} u(t)\right\|_{\infty} \int_{\mathbb{R}}\left(\partial_{x}^{2} D_{x}^{1-\frac{\alpha}{2}} u\right)^{2} \chi_{\epsilon, b}^{2} \mathrm{~d} x,
$$

By the local theory $\partial_{x} u \in L_{T}^{1} L_{x}^{\infty}$, and the integral expression will be estimated by means of Gronwall's inequality. 
By Sobolev's embedding it follows that

$$
\begin{aligned}
\left|B_{3,7,2}(t)\right| & \lesssim\|u(t)\|_{\infty} \int_{\mathbb{R}}\left(\chi_{\epsilon, b}^{2}\right)^{\prime}\left(\partial_{x}^{2} D_{x}^{1-\frac{\alpha}{2}} u\right)^{2} \mathrm{~d} x \\
& \lesssim\left(\sup _{0 \leqslant t \leqslant T}\|u(t)\|_{s_{\alpha}, 2}\right) \int_{\mathbb{R}}\left(\chi_{\epsilon, b}^{2}\right)^{\prime}\left(\partial_{x}^{2} D_{x}^{\frac{\alpha}{2}} u\right)^{2} \mathrm{~d} x
\end{aligned}
$$

and noticing that after integrating in time we obtain

$$
\int_{0}^{T}\left|B_{3,7,2}(t)\right| \mathrm{d} t \lesssim\left(\sup _{0 \leqslant t \leqslant T}\|u(t)\|_{s_{\alpha}, 2}\right) \int_{0}^{T} \int_{\mathbb{R}}\left(\chi_{\epsilon, b}^{2}\right)^{\prime}\left(\partial_{x}^{2} D_{x}^{\frac{\alpha}{2}} u\right)^{2} \mathrm{~d} x \mathrm{~d} t,
$$

where the integral expression corresponds to the $B_{1}$ term, which was already estimated in (5.9).

We conclude this step gathering the estimates corresponding to $B_{1}, B_{2}$ and $B_{3}$ combined with Gronwall's inequality and integration in time to obtain for any $\epsilon>0, b \geqslant 5 \epsilon$ and $v \geqslant 0$,

$$
\sup _{0 \leqslant t \leqslant T}\left\|\partial_{x}^{2} D_{x}^{1-\frac{\alpha}{2}} u \chi_{\epsilon, b}(\cdot+v t)\right\|_{2}^{2}+\left\|\partial_{x}^{3} u \eta_{\epsilon, b}^{2}\right\|_{L_{T}^{2} L_{x}^{2}}^{2}+\left\|\mathcal{H} \partial_{x}^{3} u \eta_{\epsilon, b}\right\|_{L_{T}^{2} L_{x}^{2}}^{2} \leqslant c_{2,2 k^{\prime}}^{*}
$$

where $c_{2,2 k+1}^{*}=c_{2,2 k+1}^{*}\left(\alpha ; \epsilon ; T ; v ;\left\|u_{0}\right\|_{s_{\alpha}, 2} ;\left\|D_{x}^{1-\frac{\alpha}{2}} \partial_{x}^{2} u_{0} \chi_{\epsilon, b}\right\|_{2}\right)>0$.

The inequality above finishes the first induction argument.

\section{$\underline{\text { CASE } m}$}

Next, our argument will combine two induction process at the same time to reach our goal.

The first induction process consists in to assume that $m$ derivatives are propagated to then prove that $m+1$ derivatives are propagated; this is summarized in the diagram below.

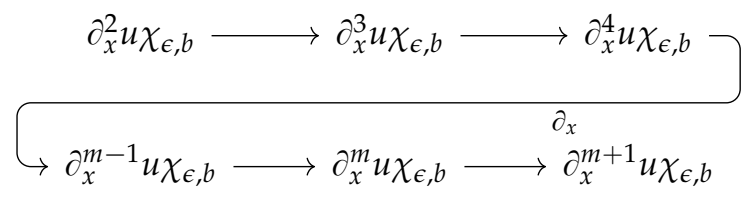

More precisely, it is described in the following figure 


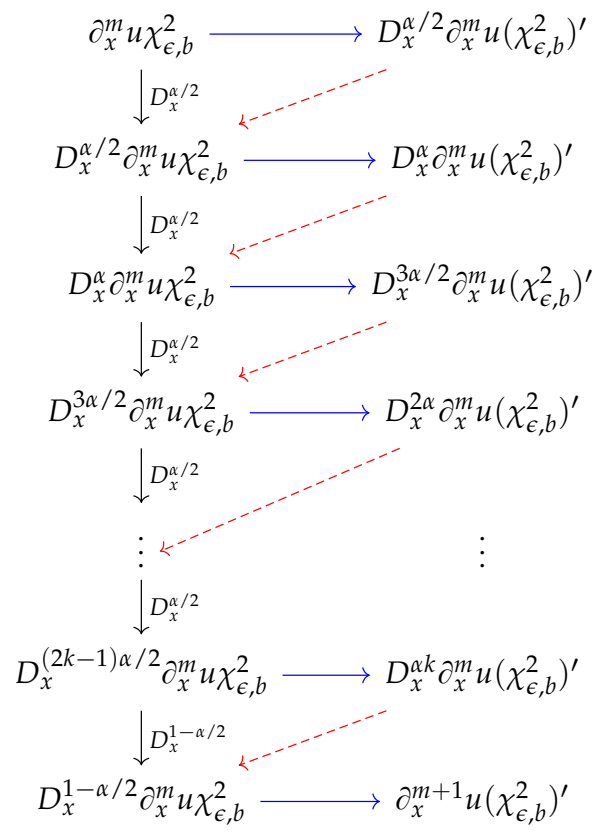

As part of this induction process, we shall assume that for any $\epsilon>0, b \geqslant 5 \epsilon, v \geqslant 0$, the following holds:

(I) for $l=2,3, \cdots, m$ and $j=0,1, \cdots, 2 k-1$

$\sup _{0 \leqslant t \leqslant T}\left\|\partial_{x}^{l} D_{x}^{\frac{\alpha j}{2}} u \chi_{\epsilon, b}(\cdot+v t)\right\|_{2}^{2}+\left\|D_{x}^{\alpha\left(\frac{j+1}{2}\right)} \partial_{x}^{l} u \eta_{\epsilon, b}\right\|_{L_{T}^{2} L_{x}^{2}}^{2}+\left\|\mathcal{H} D_{x}^{\alpha\left(\frac{j+1}{2}\right)} \partial_{x}^{l} u \eta_{\epsilon, b}^{2}\right\|_{L_{T}^{2} L_{x}^{2}}^{2} \leqslant c_{n, j}^{*}$

where $c_{l, j}^{*}=c_{l, j}^{*}\left(\alpha ; \epsilon ; T ; v ; j ;\left\|u_{0}\right\|_{s_{\alpha}, 2} ;\left\|\partial_{x}^{l} D_{x}^{\frac{\alpha j}{2}} u_{0} \chi_{\epsilon, b}\right\|_{2}\right)>0$.

(II) For $l=2,3, \cdots, m-1$

$\sup _{0 \leqslant t \leqslant T}\left\|\partial_{x}^{l} D_{x}^{1-\frac{\alpha}{2}} u \chi_{\epsilon, b}(\cdot+v t)\right\|_{2}^{2}+\left\|\partial_{x}^{l+1} u \eta_{\epsilon, b}\right\|_{L_{T}^{2} L_{x}^{2}}^{2}+\left\|\mathcal{H} \partial_{x}^{l} u \eta_{\epsilon, b}\right\|_{L_{T}^{2} L_{x}^{2}}^{2} \leqslant c_{l, 2 k}^{*}$

as usual we indicate the dependence of the parameters involved behind the constant in the right hand side above. More precisely,

$$
c_{l, j}^{*}=c_{l, j}^{*}\left(\alpha ; \epsilon ; T ; v ; j ;\left\|u_{0}\right\|_{s_{\alpha}, 2} ;\left\|\partial_{x}^{l} D_{x}^{1-\frac{\alpha}{2}} u_{0} \chi_{\epsilon, b}\right\|_{2}\right)>0 .
$$

STEP 2: 
Once our induction assumptions are fixed, we proceed as before, that is, we obtain the weighted energy estimates

$$
\begin{aligned}
& \frac{1}{2} \frac{\mathrm{d}}{\mathrm{d} t} \int_{\mathbb{R}}\left(D_{x}^{\frac{\alpha}{2}} \partial_{x}^{m} u\right)^{2} \chi_{\epsilon, b}^{2} \mathrm{~d} x \underbrace{-\frac{v}{2} \int_{\mathbb{R}}\left(D_{x}^{\frac{\alpha}{2}} \partial_{x}^{m} u\right)^{2}\left(\chi_{\epsilon, b}^{2}\right)^{\prime} \mathrm{d} x}_{B_{1}(t)} \\
& \underbrace{-\int_{\mathbb{R}}\left(D_{x}^{\frac{\alpha}{2}} \partial_{x}^{m} D_{x}^{\alpha} \partial_{x} u\right) D_{x}^{\frac{\alpha}{2}} \partial_{x}^{m} u \chi_{\epsilon, b}^{2} \mathrm{~d} x}_{B_{2}(t)}, \\
& \underbrace{+\int_{\mathbb{R}}\left(D_{x}^{\frac{\alpha}{2}} \partial_{x}^{m}\left(u \partial_{x} u\right)\right) D_{x}^{\frac{\alpha}{2}} \partial_{x}^{m} u \chi_{\epsilon, b}^{2} \mathrm{~d} x=0 .}_{B_{\mathbb{R}}(t)}
\end{aligned}
$$

$\S .1$ First, by the induction hypothesis (5.37), we take $l=m$ and $j=2 k-1$, then $\left\|B_{1}\right\|_{1}$ is bounded. More precisely,

$$
\int_{0}^{T}\left|B_{1}(t)\right| \mathrm{d} t \lesssim \int_{0}^{T} \int_{\mathbb{R}}\left(D_{x}^{\frac{\alpha}{2}} \partial_{x}^{m} u\right)^{2}\left(\chi_{\epsilon, b}^{2}\right)^{\prime} \mathrm{d} x \lesssim c_{m, 1}^{*}
$$

$\S .2$ Next, as usual in our argument to handle $B_{2}$, we first rewrite it as follows

$$
\begin{aligned}
B_{2}(t) & =\frac{1}{2} \int_{\mathbb{R}} D_{x}^{\frac{\alpha}{2}} \partial_{x}^{m} u\left[D_{x}^{\alpha} \partial_{x} ; \chi_{\epsilon, b}^{2}\right] D_{x}^{\frac{\alpha}{2}} \partial_{x}^{m} u \mathrm{~d} x \\
& =-\frac{1}{2} \int_{\mathbb{R}} D_{x}^{\frac{\alpha}{2}} \partial_{x}^{m} u\left[\mathcal{H} D_{x}^{\alpha+1} ; \chi_{\epsilon, b}^{2}\right] D_{x}^{\frac{\alpha}{2}} \partial_{x}^{m} u \mathrm{~d} x .
\end{aligned}
$$

At this point several remarks shall be made. More precisely, $B_{2}$ has different representations according the number $m$ be even or odd. The full description of these issues is presented below.

(I) If $m \in \mathbb{Q}_{1}$, then

$$
B_{2}(t)=-\frac{1}{2} \int_{\mathbb{R}} D_{x}^{m+\frac{\alpha}{2}} u\left[\mathcal{H} D_{x}^{\alpha+1} ; \chi_{\epsilon, b}^{2}\right] D_{x}^{m+\frac{\alpha}{2}} u \mathrm{~d} x .
$$

(II) If $m \in \mathbb{Q}_{2}$, then

$$
B_{2}(t)=-\frac{1}{2} \int_{\mathbb{R}} \mathcal{H} D_{x}^{m+\frac{\alpha}{2}} u\left[\mathcal{H} D_{x}^{\alpha+1} ; \chi_{\epsilon, b}^{2}\right] \mathcal{H} D_{x}^{m+\frac{\alpha}{2}} u \mathrm{~d} x
$$

We will only focus our attention on (II). Nevertheless, the way to proceed when (I) holds was described in the case $m=2$.

As was done in the previous cases, we incorporate the commutator decomposition (3.12) to obtain

$$
\begin{aligned}
B_{2}(t)= & \frac{1}{2} \int_{\mathbb{R}} \mathcal{H} D_{x}^{m+\frac{\alpha}{2}} u R_{n}(\alpha+1) \mathcal{H} D_{x}^{m+\frac{\alpha}{2}} u \mathrm{~d} x+\frac{1}{4} \int_{\mathbb{R}} \mathcal{H} D_{x}^{m+\frac{\alpha}{2}} u P_{n}(\alpha+1) \mathcal{H} D_{x}^{m+\frac{\alpha}{2}} u \mathrm{~d} x \\
& -\frac{1}{4} \int_{\mathbb{R}} D_{x}^{m+\frac{\alpha}{2}} \mathcal{H} u \mathcal{H} P_{n}(\alpha+1) \mathcal{H} D_{x}^{m+\frac{\alpha}{2}} \mathcal{H} u \mathrm{~d} x \\
= & B_{2,1}(t)+B_{2,2}(t)+B_{2,3}(t),
\end{aligned}
$$

for some positive integer $n$.

We fix $n$ satisfying

$$
2 n+1 \leqslant \alpha+1+2\left(m+\frac{\alpha}{2}\right) \leqslant 2 n+3
$$


from this we obtain $n=m$. Thus, in view of Plancherel's identity, Hölder's inequality and Proposition 3.1

$$
\begin{aligned}
\left|B_{2,1}(t)\right| & \lesssim\|\mathcal{H} u(t)\|_{2}\left\|D_{x}^{m+\frac{\alpha}{2}} R_{m}(\alpha+1) D_{x}^{m+\frac{\alpha}{2}} \mathcal{H} u(t)\right\|_{2} \\
& \lesssim\left\|u_{0}\right\|_{2}\left\|D_{x}^{2 m+2 \alpha+1}\left(\chi_{\epsilon, b}^{2}\right)\right\|_{1} .
\end{aligned}
$$

Therefore,

$$
\int_{0}^{T}\left|B_{2,1}(t)\right| \mathrm{d} t \lesssim T\left\|u_{0}\right\|_{2} .
$$

In addition to this, the terms in the decomposition (5.41) can be written as

$$
\begin{aligned}
B_{2,2}(t)= & \left(\frac{\alpha+1}{4}\right) \int_{\mathbb{R}}\left(\mathcal{H} D_{x}^{m+\alpha} u\right)^{2}\left(\chi_{\epsilon, b}^{2}\right)^{\prime} \mathrm{d} x \\
& +\left(\frac{\alpha+1}{4}\right) \sum_{d=1}^{m-1} \frac{c_{2 d+1}(-1)^{d}}{4^{d}} \int_{\mathbb{R}}\left(\mathcal{H} D_{x}^{m-d+\alpha} u\right)^{2}\left(\chi_{\epsilon, b}^{2}\right)^{(2 d+1)} \mathrm{d} x \\
& -\left(\frac{\alpha+1}{4}\right) \frac{c_{2 m+1}}{4^{m}} \int_{\mathbb{R}}\left(\mathcal{H} D_{x}^{\alpha} u\right)^{2}\left(\chi_{\epsilon, b}^{2}\right)^{(2 m+1)} \mathrm{d} x \\
= & B_{2,2,1}(t)+\sum_{d \in \mathbb{Q}_{1}(m-1)} B_{2,2, d}(t)+\sum_{d \in \mathbb{Q}_{2}(m-1)} B_{2,2, d}(t)+B_{2,2, m}(t)
\end{aligned}
$$

and

$$
\begin{aligned}
B_{2,3}(t)= & \left(\frac{\alpha+1}{4}\right) \int_{\mathbb{R}}\left(D_{x}^{m+\alpha} u\right)^{2}\left(\chi_{\epsilon, b}^{2}\right)^{\prime} \mathrm{d} x \\
& +\left(\frac{\alpha+1}{4}\right) \sum_{d=1}^{m-1} \frac{c_{2 d+1}(-1)^{d}}{4^{d}} \int_{\mathbb{R}}\left(D_{x}^{m-d+\alpha} u\right)^{2}\left(\chi_{\epsilon, b}^{2}\right)^{(2 d+1)} \mathrm{d} x \\
& -\left(\frac{\alpha+1}{4}\right) \frac{c_{2 m+1}}{4^{m}} \int_{\mathbb{R}}\left(D_{x}^{\alpha} u\right)^{2}\left(\chi_{\epsilon, b}^{2}\right)^{(2 m+1)} \mathrm{d} x \\
= & B_{2,3,1}(t)+\sum_{d \in \mathbb{Q}_{1}(m-1)} B_{2,3, d}(t)+\sum_{d \in \mathbb{Q}_{2}(m-1)} B_{2,3, d}(t)+B_{2,3, m}(t) .
\end{aligned}
$$

The terms $B_{2,2,1}$ and $B_{2,3,1}$ are positive and represent the smoothing effect after integrate in time. Besides, $\left\|B_{2,2, m}\right\|_{1}$ and $\left\|B_{2,3, m}\right\|_{1}$ are easily controlled by means of the local theory.

The remainder terms require extra arguments besides local theory to be estimated, we proceed to describe how to handle these terms.

First, for $d \in \mathbb{Q}_{1}(m-1)$

$$
\begin{aligned}
\int_{0}^{T}\left|B_{2,2, d}(t)\right| \mathrm{d} t & \lesssim \int_{0}^{T} \int_{\mathbb{R}}\left(\mathcal{H} D_{x}^{m-d+\alpha} u\right)^{2} \chi_{\epsilon / 3, b+\epsilon}^{\prime} \mathrm{d} x \mathrm{~d} t \\
& \lesssim \int_{0}^{T} \int_{\mathbb{R}}\left(\mathcal{H} D_{x}^{m-d+\alpha} u\right)^{2}\left(\chi_{\epsilon / 9, b+10 \epsilon / 9}^{2}\right)^{\prime} \mathrm{d} x \mathrm{~d} t \\
& \leqslant c_{m-d, 2}^{*}
\end{aligned}
$$


while for $d \in \mathbb{Q}_{2}(m-1)$

$$
\begin{aligned}
\int_{0}^{T}\left|B_{2,2, d}(t)\right| \mathrm{d} t & \lesssim \int_{0}^{T} \int_{\mathbb{R}}\left(\partial_{x}^{m-d} D_{x}^{\alpha} u\right)^{2} \chi_{\epsilon / 3, b+\epsilon}^{\prime} \mathrm{d} x \mathrm{~d} t \\
& \lesssim \int_{0}^{T} \int_{\mathbb{R}}\left(\partial_{x}^{m-d} D_{x}^{\alpha} u\right)^{2}\left(\chi_{\epsilon / 9, b+10 \epsilon / 9}^{2}\right)^{\prime} \mathrm{d} x \mathrm{~d} t \\
& \leqslant c_{m-d, 2}^{*} .
\end{aligned}
$$

The inequalities in (5.43) and (5.44) are obtained combining the properties of the weighted functions and (5.37).

Similar arguments can be applied to obtain

$$
\int_{0}^{T}\left|B_{2,3, d}(t)\right| \mathrm{d} t \lesssim c_{m-d, 2}^{*} \quad \text { for any } \quad d \in \mathbb{Q}_{1}(m-1) \cup \mathbb{Q}_{2}(m-1) .
$$

$\S .3$ The term $B_{3}$ is handled as in the previous steps, this is, first we decompose it according to the case. This is:

(I) if $m$ is even, then

$$
\begin{aligned}
\partial_{x}^{m} D_{x}^{\frac{\alpha}{2}}\left(u \partial_{x} u\right) \chi_{\epsilon, b}= & c_{m} D_{x}^{m+\frac{\alpha}{2}}\left(u \partial_{x} u\right) \chi_{\epsilon, b} \\
= & \frac{c_{m}}{2}\left[D_{x}^{m+\frac{\alpha}{2}} ; \chi_{\epsilon, b}\right] \partial_{x}\left(\left(u \chi_{\epsilon, b}\right)^{2}+\left(u \widetilde{\phi_{\epsilon}, b}\right)^{2}+u^{2} \psi_{\epsilon}\right) \\
& -c_{m}\left[D_{x}^{m+\frac{\alpha}{2}} ; u \chi_{\epsilon, b}\right] \partial_{x}\left(u \chi_{\epsilon, b}+u \phi_{\epsilon, b}+u \psi_{\epsilon}\right)+u \chi_{\epsilon, b} \partial_{x}^{m} D_{x}^{\frac{\alpha}{2}} \partial_{x} u \\
= & \widetilde{B_{3,1}}(t)+\widetilde{B_{3,2}}(t)+\widetilde{B_{3,3}}(t)+\widetilde{B_{3,4}}(t)+\widetilde{B_{3,5}}(t)+\widetilde{B_{3,6}}(t)+\widetilde{B_{3,7}}(t) ;
\end{aligned}
$$

(II) instead for $m$ odd,

$$
\begin{aligned}
\partial_{x}^{m} D_{x}^{\frac{\alpha}{2}}\left(u \partial_{x} u\right) \chi_{\epsilon, b}= & -c_{m} \mathcal{H} D_{x}^{m+\frac{\alpha}{2}}\left(u \partial_{x} u\right) \chi_{\epsilon, b} \\
= & \frac{c_{m}}{2}\left[\mathcal{H} D_{x}^{m+\frac{\alpha}{2}} ; \chi_{\epsilon, b}\right] \partial_{x}\left(\left(u \chi_{\epsilon, b}\right)^{2}+\left(u \widetilde{\phi_{\epsilon} b}\right)^{2}+u^{2} \psi_{\epsilon}\right) \\
& -c_{m}\left[\mathcal{H} D_{x}^{m+\frac{\alpha}{2}} ; u \chi_{\epsilon, b}\right] \partial_{x}\left(u \chi_{\epsilon, b}+u \phi_{\epsilon, b}+u \psi_{\epsilon}\right)+u \chi_{\epsilon, b} \partial_{x}^{m} D_{x}^{\frac{\alpha}{2}} \partial_{x} u \\
= & \frac{c_{m}}{2} \mathcal{H}\left[D_{x}^{m+\frac{\alpha}{2}} ; \chi_{\epsilon, b}\right] \partial_{x}\left(\left(u \chi_{\epsilon, b}\right)^{2}+\left(u \widetilde{\phi_{\epsilon, b}}\right)^{2}+u^{2} \psi_{\epsilon}\right) \\
& +\frac{c_{m}}{2}\left[\mathcal{H} ; \chi_{\epsilon, b}\right] D_{x}^{m+\frac{\alpha}{2}} \partial_{x}\left(\left(u \chi_{\epsilon, b}\right)^{2}+\left(u \widetilde{\phi_{\epsilon, b}}\right)^{2}+u^{2} \psi_{\epsilon}\right) \\
& -c_{m} \mathcal{H}\left[D_{x}^{m+\frac{\alpha}{2}} ; u \chi_{\epsilon, b}\right] \partial_{x}\left(u \chi_{\epsilon, b}+u \phi_{\epsilon, b}+u \psi_{\epsilon}\right) \\
& -c_{m}\left[\mathcal{H} ; u \chi_{\epsilon, b}\right] D_{x}^{m+\frac{\alpha}{2}} \partial_{x}\left(u \chi_{\epsilon, b}+u \phi_{\epsilon, b}+u \psi_{\epsilon}\right)+u \chi_{\epsilon, b} \partial_{x}^{m} D_{x}^{\frac{\alpha}{2}} \partial_{x} u \\
= & \widetilde{B_{3,1}}(t)+\widetilde{B_{3,2}}(t)+\widetilde{B_{3,3}}(t)+\widetilde{B_{3,4}}(t)+\widetilde{B_{3,5}}(t)+\widetilde{B_{3,6}}(t)+\widetilde{B_{3,7}}(t) \\
& +\widetilde{B_{3,8}}(t)+\widetilde{B_{3,9}}(t)+\widetilde{B_{3,10}}(t)+\widetilde{B_{3,1}}(t)+\widetilde{B_{3,12}}(t)+\widetilde{B_{3,13}}(t) .
\end{aligned}
$$

We will focus in the harder case to estimate i.e. when $m$ is odd. The case $m$ even is is simpler.

For the sake of simplicity we will gather the terms according to the tools used for its estimation without matter the order.

First, by Lemma 3.5 is clear that

$$
\left\|\widetilde{B_{3,3 l}}\right\|_{2} \lesssim\|u(t)\|_{\infty}\left\|u_{0}\right\|_{2} \quad \text { for } \quad l=1,2,3,4
$$


A combination of the Lemma 3.1, Lemma 3.3, interpolation and the Calderon's commutator estimate (3.2), give us

$$
\begin{aligned}
\left\|\widetilde{B_{3,1}}\right\|_{2} & \lesssim\|u\|_{\infty}\left(\left\|u_{0}\right\|_{2}+\left\|D_{x}^{m+\frac{\alpha}{2}}\left(u \chi_{\epsilon, b}\right)\right\|_{2}\right), \\
\left\|\widetilde{B_{3,2}}\right\|_{2} & \lesssim\|u\|_{\infty}\left(\left\|u_{0}\right\|_{2}+\left\|D_{x}^{m+\frac{\alpha}{2}}\left(u \widetilde{\phi_{\epsilon, b}}\right)\right\|_{2}\right),
\end{aligned}
$$

and

$$
\left\|\widetilde{B_{3,4+l}}\right\|_{2} \lesssim\|u\|_{\infty}\|u\|_{s_{\alpha, 2}} \text { for } \quad l \in\{0,1\}
$$

Next, applying commutator's estimate (3.9) leads to

$$
\left\|\widetilde{B_{3,7}}\right\|_{2} \lesssim\left\|\partial_{x}\left(u \chi_{\epsilon, b}\right)\right\|_{\infty}\left\|D_{x}^{m+\frac{\alpha}{2}}\left(u \chi_{\epsilon, b}\right)\right\|_{2}
$$

and

$$
\left\|\widetilde{B_{3,8}}\right\|_{2} \lesssim\left\|D_{x}^{m+\frac{\alpha}{2}}\left(u \phi_{\epsilon, b}\right)\right\|_{2}\left\|\partial_{x}\left(u \chi_{\epsilon, b}\right)\right\|_{\infty}+\left\|D_{x}^{m+\frac{\alpha}{2}}\left(u \chi_{\epsilon, b}\right)\right\|_{2}\left\|\partial_{x}\left(u \phi_{\epsilon, b}\right)\right\|_{\infty} .
$$

In order to bound the remainder terms we use (3.9) from where we get that

$$
\left\|\widetilde{B_{3,10}}\right\|_{2} \lesssim\left\|\partial_{x}\left(u \chi_{\epsilon, b}\right)\right\|_{\infty}\left\|D_{x}^{m+\frac{\alpha}{2}}\left(u \chi_{\epsilon, b}\right)\right\|_{2},
$$

and

$$
\left\|\widetilde{B_{3,11}}\right\|_{2} \lesssim\left\|\partial_{x}\left(u \chi_{\epsilon, b}\right)\right\|_{\infty}\left\|D_{x}^{m+\frac{\alpha}{2}}\left(u \phi_{\epsilon, b}\right)\right\|_{2}
$$

To finish with the estimates above, we replace $\widetilde{B_{3,13}}$ into (5.39), from that it is obtained a term which can be estimated by using integration by parts, Gronwall's inequality and the Strichartz's estimate in Theorem 1.2.

Notwithstanding all the terms above have been estimated, several issues have to be clarified. First, the non-local behavior of operator $D_{x}^{s}$ for any $s \notin 2 \mathbb{N}$ do not allow us to know where force us to localize the function $u$ and its derivatives in the places where the regularity is available.

For this reason, the term $\left\|D_{x}^{m+\frac{\alpha}{2}}\left(u \chi_{\epsilon, b}\right)\right\|_{2}$ appearing in the estimates above must be bounded, we do this by using the decomposition trick, this is

$$
D_{x}^{m+\frac{\alpha}{2}}\left(u \chi_{\epsilon, b}\right)=\chi_{\epsilon, b} D_{x}^{m+\frac{\alpha}{2}} u+\left[D_{x}^{m+\frac{\alpha}{2}} ; \chi_{\epsilon, b}\right]\left(u \chi_{\epsilon, b}+u \phi_{\epsilon, b}+u \psi_{\epsilon}\right),
$$

and if we assume that $m$ is odd the decomposition (5.53) have to be rewritten as follows

$$
\begin{aligned}
D_{x}^{m+\frac{\alpha}{2}}\left(u \chi_{\epsilon, b}\right)= & c_{m}\left[\mathcal{H} ; \chi_{\epsilon, b}\right] \partial_{x}^{m} D_{x}^{\frac{\alpha}{2}} u+c_{m} \mathcal{H}\left(\chi_{\epsilon, b} \partial_{x}^{m} D_{x}^{\frac{\alpha}{2}} u\right) \\
& +\left[D_{x}^{m+\frac{\alpha}{2}} ; \chi_{\epsilon, b}\right]\left(u \chi_{\epsilon, b}+u \phi_{\epsilon, b}+u \psi_{\epsilon}\right),
\end{aligned}
$$

where $c_{m}$ is a non-null constant. A direct application of Calderon's commutator estimate (3.2), Lemma 3.3 and interpolation imply that

$$
\begin{aligned}
\left\|D_{x}^{m+\frac{\alpha}{2}}\left(u \chi_{\epsilon, b}\right)\right\|_{2} & \lesssim\|u\|_{S_{\alpha}, 2}+\left\|\chi_{\epsilon, b} \partial_{x}^{m} D_{x}^{\frac{\alpha}{2}} u\right\|_{2}+\left\|D_{x}^{m-1+\frac{\alpha}{2}}\left(u \chi_{\epsilon, b}\right)\right\|_{2}+\left\|D_{x}^{m-1+\frac{\alpha}{2}}\left(u \phi_{\epsilon, b}\right)\right\|_{2} \\
& \lesssim\|u\|_{S_{\alpha}, 2}+\left\|\chi_{\epsilon, b} \partial_{x}^{m} D_{x}^{\frac{\alpha}{2}} u\right\|_{2}+\left\|\partial_{x}^{m}\left(u \chi_{\epsilon, b}\right)\right\|_{2}+\left\|\partial_{x}^{m}\left(u \phi_{\epsilon, b}\right)\right\|_{2} \\
& =A_{1}+A_{2}+A_{3}+A_{4} .
\end{aligned}
$$

It is straightforward to see that $A_{1}$ is bounded, this is a consequence of the local theory. The term, $A_{3}$ is handled by first noticing that

$$
\chi_{\epsilon / 5, \epsilon}(x) \chi_{\epsilon, b}(x)=\chi_{\epsilon, b}(x) \text { for all } x \in \mathbb{R},
$$


then combining Lemma 3.4 and Young's inequality

$$
\left\|\partial_{x}^{m}\left(u \chi_{\epsilon, b}\right)\right\|_{2} \lesssim\left\|\partial_{x}^{m} u \chi_{\epsilon, b}\right\|_{2}+\sum_{d=2}^{m-1} \gamma_{m, d}\left\|\partial_{x}^{d} u \chi_{\epsilon / 5, \epsilon}\right\|_{2}+\|u\|_{s_{\alpha}, 2} .
$$

Considering a slightly modification of the weights involved in (5.56) it is possible to follow the same arguments as above to estimate $A_{4}$, however, in order to avoid repetitions we will omit the calculations.

Further, after integrate in time and use the inductive hypothesis, this is (5.37), then

$$
\left\|A_{3}\right\|_{1} \lesssim \sum_{d=2}^{m} \widetilde{\gamma_{m, d}}\left(c_{d, 0}^{*}\right)^{1 / 2}+\|u\|_{L_{T}^{\infty} H_{x}^{s \alpha}} .
$$

Similarly, combining properties of the weighted functions and the induction hypothesis (5.38) produces

$$
\left\|A_{4}\right\|_{1} \lesssim \sum_{d=2}^{m} \lambda_{m, d}\left(c_{d, 2 k}^{*}\right)^{1 / 2}+\|u\|_{L_{T}^{\infty} H_{x}^{s \alpha}}
$$

Finally, to estimate $\left\|D_{x}^{m+\frac{\alpha}{2}}\left(u \phi_{\epsilon, b}\right)\right\|_{2}$ we decouple it by using Theorem 3.4 as follows

$$
\begin{aligned}
\left\|D_{x}^{m+\frac{\alpha}{2}}\left(u \phi_{\epsilon, b}\right)\right\|_{L_{T}^{2} L_{x}^{2}} \lesssim & \left\|D_{x}^{m+\frac{\alpha}{2}} \phi_{\epsilon, b}\right\|_{L_{T}^{\infty} L_{x}^{4}}\left\|u_{0}\right\|_{2}+\sum_{d \in \mathbb{Q}_{1}(m)} \frac{1}{d !}\left\|\partial_{x}^{d} \phi_{\epsilon, b} D_{x}^{m-d+\frac{\alpha}{2}} u\right\|_{L_{T}^{2} L_{x}^{2}} \\
& +\sum_{d \in \mathbb{Q}_{2}(m)} \frac{1}{d !}\left\|\partial_{x}^{d} \phi_{\epsilon, b} \mathcal{H} D_{x}^{m-d+\frac{\alpha}{2}} u\right\|_{L_{T}^{2} L_{x}^{2}} \\
\lesssim & \left\|u_{0}\right\|_{2}+\sum_{d \in \mathbb{Q}_{1}(m)} \frac{1}{d !}\left\|\varphi_{\epsilon / 8, b+\epsilon / 4} D_{x}^{m-d+\frac{\alpha}{2}} u\right\|_{L_{T}^{2} L_{x}^{2}} \\
& +\sum_{d \in \mathbb{Q}_{2}(m)} \frac{1}{d !}\left\|\varphi_{\epsilon / 8, b+\epsilon / 4} \mathcal{H} D_{x}^{m-d+\frac{\alpha}{2}} u\right\|_{L_{T}^{2} L_{x}^{2}} \\
\lesssim & \left\|u_{0}\right\|_{2}+\sum_{d \in \mathbb{Q}_{1}(m)} \frac{1}{d !}\left\|\eta_{\epsilon / 24, b+7 \epsilon / 24} D_{x}^{m-d+\frac{\alpha}{2}} u\right\|_{L_{T}^{2} L_{x}^{2}} \\
& +\sum_{d \in \mathbb{Q}_{2}(m)} \frac{1}{d !}\left\|\eta_{\epsilon / 24, b+7 \epsilon / 24} \mathcal{H} D_{x}^{m-d+\frac{\alpha}{2}} u\right\|_{L_{T}^{2} L_{x}^{2}} \\
\lesssim & \left\|u_{0}\right\|_{2}+\sum_{d=0}^{m} \frac{1}{d !}\left(c_{m-d, 1}^{*}\right)^{1 / 2}
\end{aligned}
$$

where the last inequality is a consequence of inductive hypothesis (5.37).

This step finish gathering together the estimates corresponding to $B_{1}, B_{2}$ and $B_{3}$ that combined with Gronwall's inequality and integration in time

$$
\sup _{0 \leqslant t \leqslant T}\left\|\partial_{x}^{m} D_{x}^{\frac{\alpha}{2}} u \chi_{\epsilon, b}(\cdot+v t)\right\|_{2}^{2}+\left\|D_{x}^{\alpha} \partial_{x}^{m} u \eta_{\epsilon, b}\right\|_{L_{T}^{2} L_{x}^{2}}^{2}+\left\|\mathcal{H} D_{x}^{\alpha} \partial_{x}^{m} u \eta_{\epsilon, b}\right\|_{L_{T}^{2} L_{x}^{2}}^{2} \lesssim c_{m, 2}^{*}
$$

where as usual we indicate the full dependence of the parameters behind the constant i.e. $c_{m, 2}^{*}=c_{m, 2}^{*}\left(\alpha ; \epsilon ; T ; v ;\left\|u_{0}\right\|_{s_{\alpha}, 2} ;\left\|D_{x}^{\frac{\alpha}{2}} \partial_{x}^{m} u_{0} \chi_{\epsilon, b}\right\|_{2}\right)>0$.

STEP $2 k+1:$ 
The corresponding energy weighted estimate for this step is

$$
\begin{aligned}
& \frac{1}{2} \frac{\mathrm{d}}{\mathrm{d} t} \int_{\mathbb{R}}\left(\partial_{x}^{m} D_{x}^{1-\frac{\alpha}{2}} u\right)^{2} \chi_{\epsilon, b}^{2} \mathrm{~d} x \underbrace{-\frac{v}{2} \int_{\mathbb{R}}\left(\partial_{x}^{m} D_{x}^{1-\frac{\alpha}{2}} u\right)^{2}\left(\chi_{\epsilon, b}^{2}\right)^{\prime} \mathrm{d} x}_{B_{1}(t)} \\
& \underbrace{-\int_{\mathbb{R}}\left(\partial_{x}^{m} D_{x}^{1-\frac{\alpha}{2}} D_{x}^{\alpha} \partial_{x} u\right) \partial_{x}^{m} D_{x}^{1-\frac{\alpha}{2}} u \chi_{\epsilon, b}^{2} \mathrm{~d} x}_{B_{2}(t)} \\
& +\underbrace{\int_{\mathbb{R}}\left(\partial_{x}^{m} D_{x}^{1-\frac{\alpha}{2}}\left(u \partial_{x} u\right)\right) \partial_{x}^{m} D_{x}^{1-\frac{\alpha}{2}} u \chi_{\epsilon, b}^{2} \mathrm{~d} x}_{B_{3}(t)}=0 .
\end{aligned}
$$

$\S .1$ We shall point out that the representation of $B_{1}$ may change according to the case. More precisely,

(I) If $m \in \mathbb{Q}_{1}$ then

$$
B_{1}(t)=-v \int_{\mathbb{R}}\left(D_{x}^{m+1-\frac{\alpha}{2}} u\right)^{2} \eta_{\epsilon, b}^{2} \mathrm{~d} x=-v\left\|D_{x}^{m+1-\frac{\alpha}{2}} u \eta_{\epsilon, b}\right\|_{2}^{2} .
$$

(II) If $m \in \mathbb{Q}_{2}$ then

$$
B_{1}(t)=-v \int_{\mathbb{R}}\left(\mathcal{H} D_{x}^{m+1-\frac{\alpha}{2}} u\right)^{2} \eta_{\epsilon, b}^{2} \mathrm{~d} x=-v\left\|\mathcal{H} D_{x}^{m+1-\frac{\alpha}{2}} u \eta_{\epsilon, b}\right\|_{2}^{2} .
$$

For the sake of simplicity we will study the case (I), it will be clear from the context how to proceed in the case (II).

Since

$$
D_{x}^{m+\alpha k}\left(u \eta_{\epsilon, b}\right)=D_{x}^{m+\alpha k} u \eta_{\epsilon, b}+\left[D_{x}^{m+\alpha k} ; \eta_{\epsilon, b}\right]\left(u \chi_{\epsilon, b}+u \phi_{\epsilon, b}+u \psi_{\epsilon}\right),
$$

then combining (3.9), Lemma 3.5 and interpolation

$$
\begin{aligned}
\left\|D_{x}^{m+\alpha k}\left(u \eta_{\epsilon, b}\right)\right\|_{2} & \leqslant\left\|D_{x}^{m+\alpha k} u \eta_{\epsilon, b}\right\|_{2}+\left\|\left[D_{x}^{m+\alpha k} ; \eta_{\epsilon, b}\right]\left(u \chi_{\epsilon, b}+u \phi_{\epsilon, b}+u \psi_{\epsilon}\right)\right\|_{2} \\
& \lesssim\left\|D_{x}^{m+\alpha k} u \eta_{\epsilon, b}\right\|_{2}+\left\|\partial_{x}^{m}\left(u \chi_{\epsilon, b}\right)\right\|_{2}+\left\|\partial_{x}^{m}\left(u \phi_{\epsilon, b}\right)\right\|_{2}+\left\|u_{0}\right\|_{2} .
\end{aligned}
$$

Since

$$
\chi_{\epsilon / 5, \epsilon}(x) \chi_{\epsilon, b}(x)=\chi_{\epsilon, b}(x) \text { for all } x \in \mathbb{R},
$$

then combining Lemma 3.4 and Young's inequality give us

$$
\left\|\partial_{x}^{m}\left(u \chi_{\epsilon, b}\right)\right\|_{2} \lesssim\left\|\partial_{x}^{m} u \chi_{\epsilon, b}\right\|_{2}+\sum_{d=2}^{m-1} \gamma_{m, d}\left\|\partial_{x}^{d} u \chi_{\epsilon / 5, \epsilon}\right\|_{2}+\|u\|_{s_{\alpha}, 2} .
$$

Hence, by (5.37)

$$
\left\|\partial_{x}^{m} u \chi_{\epsilon, b}\right\|_{L_{T}^{\infty} L_{x}^{2}} \lesssim\left(c_{m, 1}^{*}\right)^{1 / 2}+\sum_{d=0}^{m-1} \gamma_{m, d}\left(c_{d, 1}^{*}\right)^{1 / 2}+\|u\|_{L_{T}^{\infty} H_{x}^{s \alpha}} .
$$

and

$$
\left\|\partial_{x}^{m}\left(u \phi_{\epsilon, b}\right)\right\|_{2} \lesssim \sum_{d=2}^{m} \gamma_{m, d}\left\|\partial_{x}^{d} u \chi_{\epsilon / 20, \epsilon / 4}\right\|_{2}+\|u\|_{s_{\alpha}, 2}
$$


Analogously

$$
\left\|\partial_{x}^{m}\left(u \phi_{\epsilon, b}\right)\right\|_{L_{T}^{\infty} L_{x}^{2}} \lesssim \sum_{d=2}^{m} \gamma_{m, d}\left(c_{d, 1}^{*}\right)^{1 / 2}+\|u\|_{L_{T}^{\infty} H_{x}^{s \alpha}} .
$$

Gathering the estimated terms above allow us to obtain

$$
\left\|D_{x}^{m+\alpha k}\left(u \eta_{\epsilon, b}\right)\right\|_{L_{T}^{2} L_{x}^{2}}<\infty .
$$

Therefore, by interpolation

$$
\left\|\partial_{x}^{m} D_{x}^{1-\frac{\alpha}{2}}\left(u \eta_{\epsilon, b}\right)\right\|_{L_{T}^{2} L_{x}^{2}} \lesssim\left\|u_{0}\right\|_{2}+\left\|D_{x}^{m+\alpha k}\left(u \eta_{\epsilon, b}\right)\right\|_{L_{T}^{2} L_{x}^{2}} .
$$

With this information at hand, we can estimate the term with the regularity required, this is achieved localizing the commutator expression as follows

$$
\partial_{x}^{m} D_{x}^{1-\frac{\alpha}{2}} u \eta_{\epsilon, b}=\partial_{x}^{m} D_{x}^{1-\frac{\alpha}{2}}\left(u \eta_{\epsilon, b}\right)-\left[\partial_{x}^{m} D_{x}^{1-\frac{\alpha}{2}} ; \eta_{\epsilon, b}\right]\left(u \chi_{\epsilon, b}+u \phi_{\epsilon, b}+u \psi_{\epsilon}\right),
$$

that by using a similar argument as before we obtain

$$
\left\|\partial_{x}^{m} D_{x}^{1-\frac{\alpha}{2}} u \eta_{\epsilon, b}\right\|_{L_{T}^{2} L_{x}^{2}}<\infty .
$$

So that,

$$
\int_{0}^{T}\left|B_{1}(t)\right| \mathrm{d} t=v \int_{0}^{T}\left\|\partial_{x}^{m} D_{x}^{1-\frac{\alpha}{2}} u \eta_{\epsilon, b}\right\|_{2}^{2} \mathrm{~d} t<\infty .
$$

$\S .2$ As was evidenced at the beginning of this step, several case shall be considered. In fact, the term $B_{2}$ is represented in different ways according be the case

(I) If $m \in \mathbb{Q}_{1}$, then

$$
B_{2}(t)=-\frac{1}{2} \int_{\mathbb{R}} D_{x}^{m+1-\frac{\alpha}{2}} u\left[\mathcal{H} D_{x}^{\alpha+1} ; \chi_{\epsilon, b}^{2}\right] D_{x}^{m+1-\frac{\alpha}{2}} u \mathrm{~d} x .
$$

(II) If $m \in \mathbb{Q}_{2}$ then

$$
B_{2}(t)=-\frac{1}{2} \int_{\mathbb{R}} \mathcal{H} D_{x}^{m+1-\frac{\alpha}{2}} u\left[\mathcal{H} D_{x}^{\alpha+1} ; \chi_{\epsilon, b}^{2}\right] \mathcal{H} D_{x}^{m+1-\frac{\alpha}{2}} u \mathrm{~d} x
$$

To show how to proceed in the case that $m$ is odd, we will use the expression above and the commutator decomposition as we have previously described to obtain

$$
\begin{aligned}
B_{2}(t)= & \frac{1}{2} \int_{\mathbb{R}} \mathcal{H} D_{x}^{m+1-\frac{\alpha}{2}} u R_{n}(\alpha+1) \mathcal{H} D_{x}^{m+1-\frac{\alpha}{2}} u \mathrm{x} \\
& +\frac{1}{4} \int_{\mathbb{R}} \mathcal{H} D_{x}^{m+1-\frac{\alpha}{2}} u P_{n}(\alpha+1) \mathcal{H} D_{x}^{m+1-\frac{\alpha}{2}} u \mathrm{~d} x \\
& -\frac{1}{4} \int_{\mathbb{R}} \mathcal{H} D_{x}^{m+1-\frac{\alpha}{2}} u \mathcal{H} P_{n}(\alpha+1) \mathcal{H} D_{x}^{m+1-\frac{\alpha}{2}} \mathcal{H} u \\
= & B_{2,1}(t)+B_{2,2}(t)+B_{2,3}(t) .
\end{aligned}
$$

Is choosing $n$ according to the rule

$$
2 n+1 \leqslant \alpha+1+2\left(m+1-\frac{\alpha}{2}\right) \leqslant 2 n+3
$$

which clearly implies $n=m$. 
Next, an application of Proposition 3.1 implies that the remainder term $R_{m}(\alpha+1)$ is bounded in $L_{x}^{2}$ which let us handled $B_{2,1}$ as follows

$$
\begin{aligned}
B_{2,1}(t) & =\frac{1}{2} \int_{\mathbb{R}} \mathcal{H} D_{x}^{m+1-\frac{\alpha}{2}} u R_{m}(\alpha+1) D_{x}^{m+1-\frac{\alpha}{2}} \mathcal{H} u \mathrm{~d} x \\
& =\frac{1}{2} \int_{\mathbb{R}} \mathcal{H} u D_{x}^{m+1-\frac{\alpha}{2}} R_{m}(\alpha+1) D_{x}^{m+1-\frac{\alpha}{2}} \mathcal{H} u \mathrm{~d} x
\end{aligned}
$$

Combining Hölder's inequality and

$$
\begin{aligned}
\left|B_{2,1}(t)\right| & \lesssim\|\mathcal{H} u(t)\|_{2}\left\|D_{x}^{m+1-\frac{\alpha}{2}} R_{m}(\alpha+1) D_{x}^{m+1-\frac{\alpha}{2}} \mathcal{H} u\right\|_{2} \\
& \left.\lesssim\left\|u_{0}\right\|_{2}^{2} \| D_{x}^{2 \frac{m+3}{\left(\chi_{\epsilon}\right.}}{ }^{2}\right) \|_{1} .
\end{aligned}
$$

From this it is easy to obtain that

$$
\int_{0}^{T}\left|B_{2,1}(t)\right| \mathrm{d} t \leqslant c
$$

The control in $B_{2,1}$ allow us to fix the number of terms in $B_{2,2}$ and $B_{2,3}$.

$$
\begin{aligned}
B_{2,2}(t)= & \left(\frac{\alpha+1}{4}\right) \int_{\mathbb{R}}\left(D_{x}^{m+1} u\right)^{2}\left(\chi_{\epsilon, b}^{2}\right)^{\prime} \mathrm{d} x \\
& +\left(\frac{\alpha+1}{4}\right) \sum_{d=1}^{m-1} \frac{c_{2 d+1}(-1)^{d}}{4^{d}} \int_{\mathbb{R}}\left(D_{x}^{m+1-d} u\right)^{2}\left(\chi_{\epsilon, b}^{2}\right)^{(2 d+1)} \mathrm{d} x \\
& -\left(\frac{\alpha+1}{4}\right)\left(\frac{c_{2 m+1}}{4^{m}}\right) \int_{\mathbb{R}}\left(D_{x} u\right)^{2}\left(\chi_{\epsilon, b}^{2}\right)^{(2 m+1)} \mathrm{d} x \\
= & B_{2,2,1}(t)+\sum_{d=2}^{m-1} B_{2,2, d}(t)+B_{2,2, m+1}(t)
\end{aligned}
$$

and

$$
\begin{aligned}
B_{2,3}(t)= & \left(\frac{\alpha+1}{4}\right) \int_{\mathbb{R}}\left(\mathcal{H} D_{x}^{m+1} u\right)^{2}\left(\chi_{\epsilon, b}^{2}\right)^{\prime} \mathrm{d} x \\
& +\left(\frac{\alpha+1}{4}\right) \sum_{d=1}^{m-1} \frac{c_{2 d+1}(-1)^{d}}{4^{d}} \int_{\mathbb{R}}\left(\mathcal{H} D_{x}^{m+1-d} u\right)^{2}\left(\chi_{\epsilon, b}^{2}\right)^{(2 d+1)} \mathrm{d} x \\
& -\left(\frac{\alpha+1}{4}\right)\left(\frac{c_{2 m+1}}{4^{m}}\right) \int_{\mathbb{R}}\left(\mathcal{H} D_{x} u\right)^{2}\left(\chi_{\epsilon, b}^{2}\right)^{(2 m+1)} \mathrm{d} x \\
= & B_{2,3,1}(t)+\sum_{d=2}^{m-1} B_{2,3, d}(t)+B_{2,3, m+1}(t) .
\end{aligned}
$$

Concerning the terms $B_{2,2,1}$ and $B_{2,3,1}$, after integrate in time they grant the smoothing effect. The remainders terms in (5.70) and (5.71) have to be estimated separately.

In this sense, for $d \in\{1,2, \cdots, m-1\}$

$$
\int_{0}^{T}\left|B_{2, l+1, d}(t)\right| \mathrm{d} t \lesssim \int_{0}^{T} \int_{\mathbb{R}}\left(\mathcal{H}^{l} D_{x}^{m+1-d} u\right)^{2} \chi_{\epsilon / 3, b+\epsilon}^{\prime} \mathrm{d} x \mathrm{~d} t \quad \text { for } \quad l \in\{0,1\} .
$$

Then a combination of (5.37) and properties of the weighted functions imply that

$$
\int_{0}^{T}\left|B_{2, l+1, d}(t)\right| \mathrm{d} t \lesssim c_{m+1-d, 2 k+1}^{*} \quad \text { for any } \quad l \in\{0,1\}, \quad d=2,3, \cdots, m-2 .
$$


The cases not considered in the estimation above are handled by means of the local theory.

$\S .3$ The term $B_{2}$, can be rewritten according be the case:

(I) If $m \in \mathbb{Q}_{1}$, then there exists a non-null constant $c=c(m)$ such that

$$
\begin{aligned}
\partial_{x}^{m} D_{x}^{1-\frac{\alpha}{2}}\left(u \partial_{x} u\right) \chi_{\epsilon, b}= & c_{m}\left[D_{x}^{m+1-\frac{\alpha}{2}} ; \chi_{\epsilon, b}\right] \partial_{x}\left(\left(u \chi_{\epsilon, b}\right)^{2}+\left(u \widetilde{\phi_{\epsilon}, b}\right)^{2}+u^{2} \psi_{\epsilon}\right) \\
& +c_{m}\left[D_{x}^{m+1-\frac{\alpha}{2}} ; u \chi_{\epsilon, b}\right] \partial_{x}\left(\left(u \chi_{\epsilon, b}\right)+\left(u \phi_{\epsilon, b}\right)+\left(u \psi_{\epsilon}\right)\right) \\
& +u \chi_{\epsilon, b} \partial_{x}^{m} D_{x}^{1-\frac{\alpha}{2}} \partial_{x} u .
\end{aligned}
$$

(II) If $m \in \mathbb{Q}_{2}$ then there exists a non-null constant $c=c(m)$ such that

$$
\begin{aligned}
\partial_{x}^{m} D_{x}^{1-\frac{\alpha}{2}}\left(u \partial_{x} u\right) \chi_{\epsilon, b}= & c_{m} \mathcal{H}\left[D_{x}^{m+1-\frac{\alpha}{2}} ; \chi_{\epsilon, b}\right] \partial_{x}\left(\left(u \chi_{\epsilon, b}\right)^{2}+\left(u \widetilde{\phi_{\epsilon, b}}\right)^{2}+u^{2} \psi_{\epsilon}\right) \\
& +c_{m}\left[\mathcal{H} ; \chi_{\epsilon, b}\right] D_{x}^{m+1-\frac{\alpha}{2}} \partial_{x}\left(\left(u \chi_{\epsilon, b}\right)^{2}+\left(u \widetilde{\phi_{\epsilon, b}}\right)^{2}+u^{2} \psi_{\epsilon}\right) \\
& +c_{m} \mathcal{H}\left[D_{x}^{m+1-\frac{\alpha}{2}} ; u \chi_{\epsilon, b}\right] \partial_{x}\left(\left(u \chi_{\epsilon, b}\right)+\left(u \phi_{\epsilon, b}\right)+\left(u \psi_{\epsilon}\right)\right) \\
& +c_{m}\left[\mathcal{H} ; u \chi_{\epsilon, b}\right] D_{x}^{m+1-\frac{\alpha}{2}} \partial_{x}\left(\left(u \chi_{\epsilon, b}\right)+\left(u \phi_{\epsilon, b}\right)+\left(u \psi_{\epsilon}\right)\right) \\
& +u \chi_{\epsilon, b} \partial_{x}^{m} D_{x}^{1-\frac{\alpha}{2}} \partial_{x} u \\
= & \widetilde{B_{3,1}}(t)+\widetilde{B_{3,2}}(t)+\widetilde{B_{3,3}}(t)+\widetilde{B_{3,4}}(t)+\widetilde{B_{3,5}}(t)+\widetilde{B_{3,6}}(t) \\
& +\widetilde{B_{3,7}}(t)+\widetilde{B_{3,8}}(t)+\widetilde{B_{3,9}}(t)+\widetilde{B_{3,10}}(t)+\widetilde{B_{3,11}}(t)+\widetilde{B_{3,12}}(t) \\
& +\widetilde{B_{3,13}}(t) .
\end{aligned}
$$

To show how to proceed in the case $m$ odd we combine Lemma 3.1 and Lemma 3.3 to obtain

$$
\left\|\widetilde{B_{3,1}}\right\|_{2} \lesssim\|u\|_{\infty}\left(\left\|u_{0}\right\|_{2}+\left\|D_{x}^{m+1-\frac{\alpha}{2}}\left(u \chi_{\epsilon, b}\right)\right\|_{2}\right)
$$

and

$$
\left\|\widetilde{B_{3,2}}\right\|_{2} \lesssim\|u\|_{\infty}\left(\left\|u_{0}\right\|_{2}+\left\|D_{x}^{m+1-\frac{\alpha}{2}}\left(u \widetilde{\phi_{\epsilon}, b}\right)\right\|_{2}\right) .
$$

Since the weighted functions $\chi_{\epsilon, b}$ and $\psi_{\epsilon}$ satisfy the hypothesis of Lemma 3.5 then

$$
\left\|\widetilde{B_{3,3}}\right\|_{2} \lesssim\|u\|_{\infty}\left\|u_{0}\right\|_{2} \quad \text { for } \quad l=1,2,3,4
$$

On the other hand, the Calderon's commutator estimate (3.2) and Lemma 3.1 lead to

$$
\left\|\widetilde{B_{3,4}}\right\|_{2} \lesssim\|u\|_{1,2}\|u\|_{\infty}
$$

and

$$
\left\|\widetilde{B_{3,5}}\right\|_{2} \lesssim\|u\|_{1,2}\|u\|_{\infty} .
$$

Moreover, by the commutator estimate (3.9) we have

$$
\left\|\widetilde{B_{3,7}}\right\|_{2} \lesssim\left\|\partial_{x}\left(u \chi_{\epsilon, b}\right)\right\|_{\infty}\left\|D_{x}^{m+1-\frac{\alpha}{2}}\left(u \chi_{\epsilon, b}\right)\right\|_{2}
$$

and

$$
\left\|\widetilde{B_{3,8} \|_{2}} \lesssim\right\| D_{x}^{m+1-\frac{\alpha}{2}}\left(u \phi_{\epsilon, b}\right)\left\|_{2}\right\| \partial_{x}\left(u \chi_{\epsilon, b}\right)\left\|_{\infty}+\right\| D_{x}^{m+1-\frac{\alpha}{2}}\left(u \chi_{\epsilon, b}\right)\left\|_{2}\right\| \partial_{x}\left(u \phi_{\epsilon, b}\right) \|_{\infty} .
$$


Furthermore, an application of (3.9) yields

$$
\left\|\widetilde{B_{3,10}}\right\|_{2} \lesssim\left\|\partial_{x}\left(u \chi_{\epsilon, b}\right)\right\|_{\infty}\left\|D_{x}^{m+1-\frac{\alpha}{2}}\left(u \chi_{\epsilon, b}\right)\right\|_{2},
$$

and

$$
\left\|\widetilde{B_{3,11}}\right\|_{2} \lesssim\left\|\partial_{x}\left(u \chi_{\epsilon, b}\right)\right\|_{\infty}\left\|D_{x}^{m+1-\frac{\alpha}{2}}\left(u \phi_{\epsilon, b}\right)\right\|_{2} .
$$

Notice that in the inequalities (5.72)-(5.73) and (5.74)-(5.77) there are several terms which have been not estimated yet.

Firstly,

$$
\begin{aligned}
D_{x}^{m+1-\frac{\alpha}{2}}\left(u \chi_{\epsilon, b}\right)= & c_{m} \partial_{x}^{m} \mathcal{H} D_{x}^{1-\frac{\alpha}{2}} u \chi_{\epsilon, b}+\left[D_{x}^{m+1-\frac{\alpha}{2}} ; \chi_{\epsilon, b}\right]\left(u \chi_{\epsilon, b}+u \phi_{\epsilon, b}+u \psi_{\epsilon}\right) \\
= & \mathcal{H}\left(\chi_{\epsilon, b} \partial_{x}^{m} D_{x}^{1-\frac{\alpha}{2}} u\right)-\left[\mathcal{H} ; \chi_{\epsilon, b}\right] \partial_{x}^{m} D_{x}^{1-\frac{\alpha}{2}} u \\
& +\left[D_{x}^{m+1-\frac{\alpha}{2}} ; \chi_{\epsilon, b}\right]\left(u \chi_{\epsilon, b}+u \phi_{\epsilon, b}+u \psi_{\epsilon}\right)
\end{aligned}
$$

where $c_{m}$ is a non-null constant.

Since the arguments to estimate the expression on the right have been previously used, we will only indicate the tools used.

Combining the Calderon's commutator estimate (3.2), (3.9) and interpolation imply that

$$
\left\|D_{x}^{m+1-\frac{\alpha}{2}}\left(u \chi_{\epsilon, b}\right)\right\|_{2} \lesssim\left\|\partial_{x}^{m} D_{x}^{1-\frac{\alpha}{2}} u \chi_{\epsilon, b}\right\|_{2}+\|u\|_{\mathcal{S}_{\alpha}, 2}+\left\|\partial_{x}^{m}\left(u \chi_{\epsilon, b}\right)\right\|_{2}+\left\|\partial_{x}^{m}\left(u \phi_{\epsilon, b}\right)\right\|_{m} .
$$

Notice that the first term on the right hand side is the quantity to be estimated. The third and fourth term are handled by using similar arguments as those described in (5.61)-(5.65).

Next, the condition

$$
\alpha \in\left[\frac{2}{2 k+1}, \frac{1}{k}\right) \Rightarrow m+1-\frac{\alpha}{2} \leqslant m+\alpha k
$$

is used to obtain information from the previous cases. This is achieved combining Theorem 3.4 and (5.37), that is,

$$
\begin{aligned}
\left\|D_{x}^{m+\alpha k}\left(u \phi_{\epsilon, b}\right)\right\|_{L_{T}^{2} L_{x}^{2}} \lesssim & \left\|D_{x}^{m+\alpha k} \phi_{\epsilon, b}\right\|_{L_{T}^{\infty} L_{x}^{4}}\left\|u_{0}\right\|_{2}+\sum_{d \in \mathbb{Q}_{1}(m)} \frac{1}{d !}\left\|\partial_{x}^{d} \phi_{\epsilon, b} D_{x}^{m+\alpha k-d} u\right\|_{L_{T}^{2} L_{x}^{2}} \\
& +\sum_{d \in \mathbb{Q}_{2}(m)} \frac{1}{d !}\left\|\partial_{x}^{d} \phi_{\epsilon, b} \mathcal{H} D_{x}^{m+\alpha k-d} u\right\|_{L_{T}^{2} L_{x}^{2}} \\
\lesssim & \left\|u_{0}\right\|_{2}+\sum_{d \in \mathbb{Q}_{1}(m)} \frac{1}{d !}\left\|\varphi_{\epsilon / 8, b+\epsilon / 4} D_{x}^{m+\alpha k-d} u\right\|_{L_{T}^{2} L_{x}^{2}} \\
& +\sum_{d \in \mathbb{Q}_{2}(m)} \frac{1}{d !}\left\|\varphi_{\epsilon / 8, b+\epsilon / 4} \mathcal{H} D_{x}^{m+\alpha k-d} u\right\|_{L_{T}^{2} L_{x}^{2}} \\
\lesssim & \left\|u_{0}\right\|_{2}+\sum_{d \in \mathbb{Q}_{1}(m)} \frac{1}{d !}\left\|\eta_{\epsilon / 24, b+7 \epsilon / 24} D_{x}^{m+\alpha k-d} u\right\|_{L_{T}^{2} L_{x}^{2}} \\
& +\sum_{d \in \mathbb{Q}_{2}(m)} \frac{1}{d !}\left\|\eta_{\epsilon / 24, b+7 \epsilon / 24} \mathcal{H} D_{x}^{m+\alpha k-d} u\right\|_{L_{T}^{2} L_{x}^{2}} \\
\lesssim & \left\|u_{0}\right\|_{2}+\sum_{d=0}^{m} \frac{1}{d !}\left(c_{m+\alpha k-d, 2 k-1}^{*}\right)^{1 / 2} .
\end{aligned}
$$


So that interpolation provide the bound

$$
\left\|\partial_{x}^{m} D_{x}^{1-\frac{\alpha}{2}}\left(u \phi_{\epsilon, b}\right)\right\|_{L_{T}^{2} L_{x}^{2}} \lesssim\left\|u_{0}\right\|_{2}+\left\|D_{x}^{m+\alpha k}\left(u \phi_{\epsilon, b}\right)\right\|_{L_{T}^{2} L_{x}^{2}} .
$$

Analogously is estimated $\left\|\partial_{x}^{m} D_{x}^{1-\frac{\alpha}{2}}\left(u \widetilde{\phi_{\epsilon, b}}\right)\right\|_{L_{T}^{2} L_{x}^{2}}$.

Inserting $\widetilde{B_{3,13}}$ into (5.60) it is obtained a term which can be estimated by integration by parts, Gronwall's inequality and the Strichartz's estimate in Theorem 1.2, i.e $\partial_{x} u \in$ $L^{1}\left([0, T]: L^{\infty}(\mathbb{R})\right)$.

Finally, gathering together the estimates corresponding to this step combined with Gronwall's inequality and integration in time, we obtain the desired estimate, this is, for any $\epsilon>0, b \geqslant 5 \epsilon$ and $v \geqslant 0$,

$$
\sup _{0 \leqslant t \leqslant T}\left\|\partial_{x}^{m} D_{x}^{1-\frac{\alpha}{2}} u \chi_{\epsilon, b}(\cdot+v t)\right\|_{2}^{2}+\left\|\partial_{x}^{m+1} u \eta_{\epsilon, b}\right\|_{L_{T}^{2} L_{x}^{2}}^{2}+\left\|\mathcal{H} \partial_{x}^{m+1} u \eta_{\epsilon, b}\right\|_{L_{T}^{2} L_{x}^{2}}^{2} \lesssim c_{m, 2 k+1}^{*},
$$

where $c_{m, 2 k+1}^{*}=c_{m, 2 k+1}^{*}\left(\alpha ; k ; \epsilon ; T ; v ;\left\|u_{0}\right\|_{s_{\alpha}, 2} ;\left\|D_{x}^{1-\frac{\alpha}{2}} \partial_{x}^{m} u_{0} \chi_{\epsilon, b}\right\|_{2}\right)>0$.

This estimate finish the 2 -induction process.

Throughout the proof we have always assumed as much regularity as possible on the function $u$, nevertheless the way to proceed in the general case involves the device of regularization of Bona, Smith [4].

More precisely, we consider initial data $u_{0} \in H^{s_{\alpha}^{+}}(\mathbb{R})$, then we regularize the initial data $u_{0}^{\mu}=\rho_{\mu} * u_{0}$, where $\rho$ is a positive smooth function with compact support, more precisely $\rho \in C_{0}^{\infty}(\mathbb{R})$ with $\operatorname{supp}(\rho) \subset(-1,1)$ and $\|\rho\|_{1}=1$.

For $\mu>0$ we define the family

$$
\rho_{\mu}(x)=\mu^{-1} \rho\left(\frac{x}{\mu}\right) \quad x \in \mathbb{R} .
$$

The solution $u^{\mu}$ associated to the IVP (1.1) with initial data $u_{0}^{\mu}$ satisfies

$$
u^{\mu} \in C\left([0, T]: H^{\infty}(\mathbb{R})\right) \text {. }
$$

Applying the results obtained in the section of the 2-inductive process to the function $u^{\mu}$ allows us conclude that, for any $\epsilon>0, b \geqslant 5 \epsilon, v \geqslant 0$, the following holds:

(a) for $l=2,3, \cdots, m$ and $j=0,1, \cdots, 2 k-1$

$$
\begin{aligned}
& \sup _{0 \leqslant t \leqslant T}\left\|\partial_{x}^{l} D_{x}^{\frac{\alpha j}{2}} u^{\mu} \chi_{\epsilon, b}(\cdot+v t)\right\|_{2}^{2}+\left\|\partial_{x}^{l} D_{x}^{\alpha\left(\frac{j+1}{2}\right)} u^{\mu} \eta_{\epsilon, b}\right\|_{L_{T}^{2} L_{x}^{2}}^{2} \\
& +\left\|\partial_{x}^{l} \mathcal{H} D_{x}^{\alpha\left(\frac{j+1}{2}\right)} u^{\mu} \eta_{\epsilon, b}\right\|_{L_{T}^{2} L_{x}^{2}}^{2} \leqslant c_{l, j}^{*}
\end{aligned}
$$

where $c_{l, j}^{*}=c_{l, j}^{*}\left(\alpha ; k ; \epsilon ; T ; v ; l ; j ;\left\|u_{0}^{\mu}\right\|_{s_{\alpha}, 2} ;\left\|\partial_{x}^{l} D_{x}^{\frac{\alpha j}{2}} u_{0}^{\mu} \chi_{\epsilon, b}\right\|_{2}\right)>0 ;$

(b) for $l=2,3, \cdots, m$

$$
\begin{aligned}
& \sup _{0 \leqslant t \leqslant T}\left\|\partial_{x}^{l} D_{x}^{1-\frac{\alpha}{2}} u^{\mu}(t) \chi_{\epsilon, b}(\cdot+v t)\right\|_{2}+\left\|\partial_{x}^{l+1} u^{\mu} \eta_{\epsilon, b}\right\|_{L_{T}^{2} L_{x}^{2}}^{2} \\
& \quad+\left\|\mathcal{H} \partial_{x}^{l+1} u^{\mu} \eta_{\epsilon, b}\right\|_{L_{T}^{2} L_{x}^{2}}^{2} \leqslant c_{l, 2 k+1}^{*},
\end{aligned}
$$

where $c_{l, 2 k+1}^{*}=c_{l, 2 k+1}^{*}\left(\alpha ; k ; \epsilon ; T ; v ; n ;\left\|u_{0}^{\mu}\right\|_{s_{\alpha}, 2} ;\left\|\partial_{x}^{n} D_{x}^{1-\frac{\alpha}{2}} \partial_{x}^{2} u_{0}^{\mu} \chi_{\epsilon, b}\right\|_{2}\right)>0$. 
Next, we prove that the constants $c_{l, j}^{*}$ and $c_{l, 2 k+1}^{*}$ are independent of the parameter $\mu$.

First notice that

$$
\left\|u_{0}^{\mu}\right\|_{s_{\alpha, 2}} \leqslant\left\|u_{0}\right\|_{s_{\alpha}, 2}\left\|\widehat{\rho_{\mu}}\right\|_{\infty} \leqslant\left\|u_{0}\right\|_{s_{\alpha}, 2} .
$$

Next, the weighted function $\chi_{\epsilon, b}(x)=0$ for $x \leqslant \epsilon$, therefore when we restrict $\mu \in(0, \epsilon)$ it follows by Young's inequality that

$$
\begin{aligned}
\int_{\epsilon}^{\infty}\left(\partial_{x}^{l} D_{x}^{\frac{\alpha j}{2}} u_{0}^{\mu}\right)^{2} \mathrm{~d} x & =\int_{\epsilon}^{\infty}\left(\rho_{\mu} * \partial_{x}^{l} D_{x}^{\frac{\alpha j}{2}} u_{0} \mathbb{1}_{[0, \infty)}\right)^{2} \mathrm{~d} x \\
& \leqslant\left\|\rho_{\mu}\right\|_{1}\left\|\partial_{x}^{l} D_{x}^{\frac{\alpha j}{2}} u_{0}\right\|_{L^{2}((0, \infty))} \\
& =\left\|\partial_{x}^{l} D_{x}^{\frac{\alpha j}{2}} u_{0}\right\|_{L^{2}((0, \infty))},
\end{aligned}
$$

$l=2,3, \cdots, m$ and $j=0,1, \cdots, 2 k-1$.

Similarly can be proved that for $\mu \in(0, \epsilon)$ the following inequality holds

$$
\left\|\partial_{x}^{l} D_{x}^{1-\frac{\alpha}{2}} \partial_{x}^{2} u_{0}^{\mu} \chi_{\epsilon, b}\right\|_{2}^{2} \leqslant\left\|\partial_{x}^{l} D_{x}^{1-\frac{\alpha}{2}} \partial_{x}^{2} u_{0}\right\|_{L^{2}((0, \infty))} \quad \text { for } \quad l=2,3, \cdots, m .
$$

Also, as part of the argument, the continuous dependence upon the initial data is used, this is

$$
\sup _{0 \leqslant t \leqslant T}\left\|u^{\mu}(t)-u(t)\right\|_{\mathcal{S}_{\alpha}, 2} \underset{\mu \rightarrow 0}{\longrightarrow} 0 .
$$

The proof finish combining the remarks underlined above, the independence of the constants $c_{l, j}^{*}$ and $c_{l, 2 k+1}^{*}$, of the parameter $\mu$, these joint with a weak compactness argument and Fatou's Lemma allow to conclude the proof.

\section{AcKnowledgments}

The results of this paper are part of the author's Ph.D dissertation at IMPA-Brazil. He gratefully acknowledges the encouragement and assistance of his advisor, Prof. F. Linares. He also express appreciation for the careful reading of the manuscript done by O. Riaño.

\section{REFERENCES}

[1] B. Bajšanski, R. Coifman, On singular integrals, in: Proc. Sympos. Pure Math. Chicago, Amer. Math. Soc. Providence, RI, 1966, 1-17.

[2] Á. BÉNYI, T. OH, Smoothing of commutators for a Hörmander class of bilinear pseudodifferential operators, J. Fourier Anal. Appl.20 (2014), no.2, 282-300.

[3] J. Berg, J. LöftröM, Interpolation Spaces, Springer-Verlag, 1976.

[4] J. L. Bona, R. Smith, The initial value problem for the Korteweg-de Vries equation, Philos. Trans.R. Soc. London. Ser. A,278 (1975), 555-601.

[5] J. Bourgain And D. Li, On an endpoint Kato-Ponce inequality. Differential Integral Equations (11/12) 27 (2014c), 1037-1072.

[6] A.P. CAlderon, Commutators of singular integral operators, Proc. Natl. Acad. Sci. USA 53 (1965).

[7] R. COIFMAN, V.Wickerhauser, The scattering transform for the Benjamin-Ono equation, Inverse problems, 6(1990), 825-861.

[8] L. Dawson, H. McGahagan and G. Ponce, On the decay properties of solutions to a class of Schrödinger equations, Proc. Amer. Math. Soc. 136 (2008), no.6, 2081-2090.

[9] G. Folland, Introduction to Partial Differential Equations second edition, Princeton University Press, 1995.

[10] R.L. Frank, E. Lenzmann, Uniqueness of non-linear ground states for fractional Laplacians in $\mathbb{R}$, Acta Math. 210 261-318.

[11] J. Ginibre And G. Velo, Commutator expansions and smoothing properties of generalized Benjamin-Ono equations, Ann. Inst. H. Poincaré Phys. Théorique, 51 (1989), 221-229.

[12] J. Ginibre and G. Velo, Smoothing properties and existence of solutions for the generalized Benjamin-Ono equations, J. Differential Equations, 93 (1991), 150-212.

[13] L. Grafakos, Classical Fourier Analysis Second Edition, Springer, New York, (2008). 
[14] P. Isaza, F. Linares, G. Ponce, On the propagation of regularity and decay of solutions to the $k$-generalized Korteweg-de Vries equation, Comm. Partial Differential Equations 40 (2015), 1336-1364.

[15] P. Isaza, F. Linares, G. Ponce, On the propagation of regularities in solutions of the Benjamin-Ono equation, J. Funct. Anal.(270) (2016) 976- 1000.

[16] P. Isaza, F. Linares, G. Ponce, On the propagation of regularity of solutions of the Kadomtsev-Petviashvili equation, SIAM J. Math. Anal., 48, no. 2, 1006-1024, 2016.

[17] T. Kato, On the Cauchy problem for the (generalized) Korteweg-de Vries equations, Advances in Mathematics Supplementary Studies, Stud. Math. 8 (1983) 93-128.

[18] T. Kato, Quasilinear equations of evolution, with applications to partial differential equations, Lectures Notes in Math., vol 448, Springer -Verlag, Berlin and New York, 1975, 27-50. +

[19] T. Kato, G. Ponce, Commutator estimates and the Euler and Navier-Stokes equations, Comm Pure Appl. Math 41 (1988) 891-907.

[20] C.E. Kenig, F. Linares, G. Ponce, L. VeGa, On the regularity of solutions to the $k$-generalized Korteweg-de Vries equation, (2016). arXiv:1606.03715v2.

[21] C.E. Kenig, G. Ponce, L. Vega, Well-posedness and scattering results for the generalized Korteweg-de Vries equation via contraction principle. Comm. Pure Appl. Math 46 (1993) 527-620.

[22] C.E. Kenig, G. Ponce, L. Vega, On the generalized Benjamin-Ono equation, Trans . Amer. Math.Soc. 342 (1994), 155-172.

[23] C.E. Kenig, G. Ponce, and L. Vega Well-posedness of the initial value problem for the Korteweg-de Vries equation, J.Amer. Math. Soc., 4 (1991), 323-346.

[24] C. Kenig, G. Ponce, L. Vega, Oscillatory integrals and regularity of dispersive equations, Indiana Univ. Math. J.,40 (1991), 33-69.

[25] C. KLEIN, J.C. SAUt, A numerical approach to blow-up issues for dispersive perturbations of Burgers equation, Physica D 295-296 (2015), 46-65. 38.

[26] R.L. Frank, E. Lenzmann, Uniqueness of non-linear ground states for fractional Laplacians in $\mathbb{R}$, Acta Math. 210 261-318.

[27] D. Li, On Kato-Ponce and fractional Leibniz, (2016) arXiv:1609.01780v2.

[28] F. Linares, G. Ponce, Introduction to Nonlinear Dispersive Equations second edition, Springer, New York, 2015.

[29] F. Linares, G. Ponce, On special regularity properties of solutions of the Zakharov-Kuznetsov equation, Commun. Pure Appl. Anal. 17 (2018), no. 4, 1561-1572.

[30] F. Linares, D. PILOD, J.C. SAut, Remarks on the orbital stability of ground state solutions of fKdV and related equations, Adv. Differ. Equ. 20 835-58.

[31] F. Linares, D. Pilod and J.C. Saut, Dispersive perturbations of Burgers and hyperbolic equations I: Local theory, SIAM J.Math. Anal. Vol 46,No 2, pp.1505-1537.

[32] L. Molinet, S. Vento, Improvement of the energy method for strongly non resonant dispersive equations and applications, Anal. PDE., 8 (2015), 1455-1496.

[33] L. Molinet, D. Pilod, S. Vento, On well-posedness for some dispersive perturbations of Burger's equation, arXiv: 1702.03191v2.

[34] A.J. Mendez, On the propagation of regularity for solutions of the dispersion generalized Benjamin-Ono equation, (2018) arXiv:1901.00823v1.

[35] PAVA. J, Stability properties of solitary waves for fractional KdV and BBM equations, Nonlinearity 31 (2018), no. 3, 920-956.

[36] E.M. Stein, Singular Integrals and Differentiability Properties of Functions, Princeton University Press, Princeton, 1970.

Instituto Nacional de Matematica Pura e Aplicada, Rio de Janeiro, RJ, Brasil

E-mail address: amendez@impa.br 